\title{
Exponential Amplification by Redox Cross-Catalysis and Unmasking of Doubly Protected Molecular Probes
}

\author{
Justine Pallu, Charlie Rabin, Pan Hui, Thamires S. Moreira, Geordie Creste, ${ }^{\dagger}$ Corentin Calvet, \\ Benoît Limoges, ${ }^{*}$ François Mavré, ${ }^{*}$ and Mathieu Branca*
}

Université de Paris, Laboratoire d'Electrochimie Moléculaire, UMR 7591, CNRS, F-75013 Paris, France

KEYWORDS: autocatalysis, cross-activation, exponential amplification, redox cycling, autoxidation, naphthoquinone, hydrogen peroxide, molecular probe, chemosensor, aromatic boronate

\begin{abstract}
The strength of autocatalytic reactions lies in their ability to provide a powerful means of molecular amplification, which can be very useful for improving the analytical performances of a multitude of analytical and bioanalytical methods. However, one of the major difficulties in designing an efficient autocatalytic amplification system is the requirement for reactants that are both highly reactive and chemically stable in order to avoid limitations imposed by undesirable background amplifications. In the present work, we devised a reaction network based on a redox cross-catalysis principle, in which two catalytic loops activate each other. The first loop, catalyzed by $\mathrm{H}_{2} \mathrm{O}_{2}$, involves the oxidative deprotection of a naphthylboronate ester probe into a redox-active naphthohydroquinone, which in turn catalyzes the production of $\mathrm{H}_{2} \mathrm{O}_{2}$ by redox cycling in the presence of a reducing enzyme/substrate couple. We present here a set of new molecular probes with improved reactivity and stability, resulting in particularly steep sigmoidal kinetic traces and enhanced discrimination between specific and nonspecific responses. This translates into the sensitive detection of $\mathrm{H}_{2} \mathrm{O}_{2}$ down to a few nM in less than 10 minutes or a redox cycling compound such as the 2-amino-3-chloro-1,4-naphthoquinone $\mathrm{H}_{2} \mathrm{O}_{2}$ down to 50 pM in less than 30 minutes. The critical reason leading to these remarkably good performances is the extended stability stemming from the double masking of the naphthohydroquinone core by two boronate groups, a counterintuitive strategy if we consider the need for two equivalents of $\mathrm{H}_{2} \mathrm{O}_{2}$ for full deprotection. An in-depth study of the mechanism and dynamics of this complex reaction network is conducted in order to better understand, predict and optimize its functioning. From this investigation, the time response as well as detection limit are found highly dependent on $\mathrm{pH}$, nature of buffer, and concentration of the reducing enzyme.
\end{abstract}

\section{Introduction}

In the context of chemical or biochemical analysis, it is common to rely on molecular or biomolecular amplifications to improve the analytical performances of a detection method (i.e., sensitivity, limit of detection (LOD), analysis time, dynamic range of concentration, ...). ${ }^{1,2}$ This is even more important when the targeted molecules or biomolecules are present at low concentrations. In most cases, molecular amplifications result from catalytic reactions wherein a molecule (either the analyte acting as a catalyst or a label catalyst revealing indirectly the presence of the analyte) triggers the catalysis of a reaction capable to generate a detectable signal (which can be optical, electrochemical, thermal, gravimetric, etc ...). Under these conditions, the product linearly accumulates with time and the amplification factor depends only on the catalytic rate and reaction time. ${ }^{3}$ A popular example is the signal amplification resulting from the enzyme label involved in enzymelinked immunosorbent assays.4, 5 However, greater molec- ular amplifications can be achieved by adequately combining the reactivity of different catalysts. One possibility is to cascade the reactivity of two catalysts, wherein a first one catalyzes the production of a second one. In such situation, the product of the secondary catalytic reaction accumulates quadratically with time. ${ }^{6-11} \mathrm{An}$ even more powerful approach is to produce an exponential signal gain from an autocatalytic reaction, for which one of the products catalyzes its own formation. ${ }^{12-14} \mathrm{~A}$ well-known example is the polymerase chain reaction, which through self-replication of a targeted DNA sequence allows the ultimate detection of a few molecules of nucleic acids in a few tens of microliters. ${ }^{15}$ While autocatalytic replication is well suited to nucleic acids, ${ }^{16-19}$ it is much more challenging to design for other molecules. ${ }^{13}$ Among the few examples of molecular self-replication strategies developed to date for the detection of small molecules is the dendritic chain reaction (DCR) first described in 2009 by Shabat and his collaborators. $^{20,}{ }^{21}$ In this concept, the analyte triggers its self-replication through the decomposition of a dendritic molecular 
probe containing at least two masked equivalents of the analyte. This methodology was applied to the autocatalytic detection of $\mathrm{H}_{2} \mathrm{O}^{20,22,23}$ and fluoride ions. ${ }^{24-27}$ More recently, alternative strategies based on self-propagating thiolatedisulfide exchange, ${ }^{28,} 29$ methionase chain reaction, ${ }^{30}$ or photoinduced autocatalysis ${ }^{31,32}$ were also reported. However, all these methods suffer from undesirable background reactions, which prevent the achievement of very low analyte concentrations. The main reason of these background reactions is the intrinsic lack of stability of starting coreactants, leading to self-initiation of the amplification cycle in the absence of analyte. Therefore, a critical issue is to design systems involving coreactants with high chemical stability and at the same time high reactivity and selectivity towards the trigger of the reaction. This is certainly the major challenge facing the exponential signal amplification strategies. 33 An additional drawback is the slowness of the approaches proposed to date, sometimes requiring waiting several hours for an analytical response. ${ }^{21,23}$

To address these drawbacks and contribute to the development of alternative solutions, we recently proposed a new versatile molecular-based autocatalytic system ${ }^{34,35}$ in which the exponential gain is generated from cross-activation of two catalytic loops, as depicted in Scheme 1A. The first loop (Loop 1 ) is based on a $\mathrm{H}_{2} \mathrm{O}_{2}$-mediated deprotection of a boronate ester probe $(\mathrm{P})$ to a hydroquinone derivative $\left(\mathrm{QH}_{2}\right)$, which, in the presence of $\mathrm{O}_{2}$ in solution, spontaneously autoxidizes to a quinone $(\mathrm{Q})$ with the concomitant regeneration of $\mathrm{H}_{2} \mathrm{O}_{2}$ (the latter acting thus as a catalyst in Loop 1). The autocatalysis is then engendered from the back conversion of $\mathrm{Q}$ into its reduced $\mathrm{QH}_{2}$ form in the presence of a reducing substrate $S$ (or alternatively an enzyme/reducing substrate couple E/S), thus forming the second catalytic loop (Loop 2, which is also a redox cycle wherein $\mathrm{Q} / \mathrm{QH}_{2}$ serves as a redox catalyst). Analytical derivations as well as numerical simulations of the kinetics of this redox cross-catalytic system allowed us to demonstrate the overstoichiometric production of $\mathrm{H}_{2} \mathrm{O}_{2}$ coupled with the exponential decrease of probe and substrate concentrations with time. ${ }^{34,36}$ This autocatalytic set is conceptually different from the previously reported branchedchain or self-propagating amplification reactions in that it does not require the design of a dendritic probe capable of releasing two or more equivalents of the trigger by self-immolation. Another advantage is that it offers the possibility to trigger the reaction either by $\mathrm{H}_{2} \mathrm{O}_{2}$ or any redox cycling compound, thus providing an extended degree of freedom to couple this molecular amplification not only to the detection of $\mathrm{H}_{2} \mathrm{O}_{2}$ (directly or indirect produced) but also to the detection of molecules exhibiting redox cycling properties (also either directly or indirectly generated).

Using the boronate ester probe $\mathbf{P}_{\mathbf{1}}$ shown in Scheme ${ }_{1} \mathrm{~B}$, detection of submicromolar or subnanomolar concentrations of $\mathrm{H}_{2} \mathrm{O}_{2}$ or a naphthoquinone, respectively, within 1 hr was demonstrated. ${ }^{34}$ Although these analytical performances are significantly better than those previously achieved for $\mathrm{H}_{2} \mathrm{O}_{2}$ by DCR, ${ }^{20,} 22,23,37,3^{8}$ they are still limited by the lack of chemical stability of $\mathbf{P}_{\mathbf{1}}$, which in the absence of the trigger tends to slowly self-deprotect into a naphthohydroquinone. This lack of stability turns out to be intrinsic to the self-immolating spacer installed on the probe, ${ }^{34} \mathrm{a}$ recurrent issue of molecular probes based on self-immolation and which is at the origin of the unsatisfactory analytical performances encountered so far. To access more robust and stable probes, it is thus important to design probes that no longer rely on self-immolation. This is what we address here with the new boronate-based probes shown in Scheme $1 \mathrm{C}$. Instead of introducing the masking boronate ester function via a remote self-immolating spacer, we have chosen to place it directly on the naphthyl ring, leading to either the single-protected naphthol monoboronate ester probe $\mathbf{P}_{2}$ or the doubly protected naphthyl diboronate ester probes $\mathbf{P}_{3}$ and $\mathbf{P}_{4}$. By reaction with $\mathrm{H}_{2} \mathrm{O}_{2}$, these probes are expected to release a 1,4-naphthohydroquinone $\left(1,4-\mathrm{NQH}_{2}\right)$, which in the presence of $\mathrm{O}_{2}$ spontaneously autoxidizes into 1,4-naphthoquinone $\left(1,4^{-}\right.$ NQ) with concomitant regeneration of $\mathrm{H}_{2} \mathrm{O}_{2}$, as previously shown with $\mathbf{P}_{1}{ }^{34}$ Another important feature we have considered here is to design boronate probes with an improved reactivity towards $\mathrm{H}_{2} \mathrm{O}_{2}$ as well as an optimized redox cycling capability, with the underlying idea to outcompete the background reactions..$^{36}$

Scheme 1. (A) General principle of the autocatalytic reaction scheme based on cross-activation of two catalytic loops. (B) Boronate-based probe previously used in ref 34. This probe is converted by $\mathrm{H}_{2} \mathrm{O}_{2}$ in an intermediate phenolic compound that self-immolates into a quinone methide and a 1,4-naphthohydroquinone. (C) The new probes used in the present study.

A

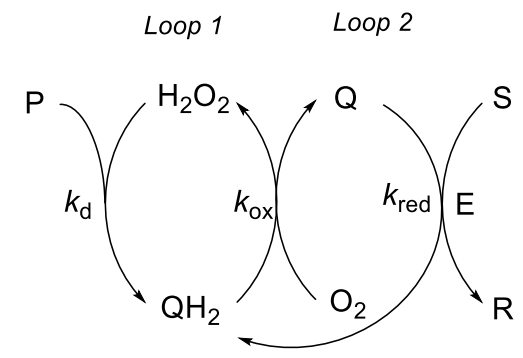

B previous work

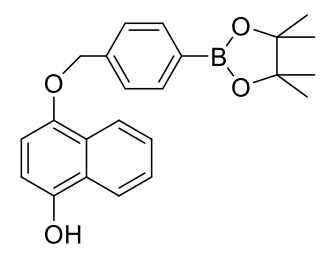

C this work<smiles>CC1(C)OB(c2ccc(O)c3ccccc23)OC1(C)C</smiles>

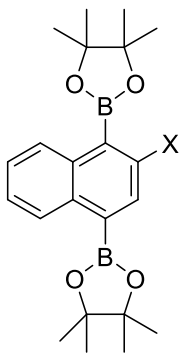

$P_{1}$
$P_{3}: X=-H$

$P_{4}: X=-O M e$ 
As it will be shown, the single-protected probe $\mathbf{P}_{\mathbf{2}}$ generates a remarkably efficient autocatalytic reaction (LOD of o.1 $\mu \mathrm{M} \mathrm{H}_{2} \mathrm{O}_{2}$ in less than 10 min at $\mathrm{pH} 8.5$ ) thanks to a fast $\mathrm{H}_{2} \mathrm{O}_{2}$-mediated deprotection rate $\left(k_{d}\right)$ (faster than $\mathbf{P}_{\mathbf{1}}$ ). However, the lack of chemical stability of $\mathbf{P}_{\mathbf{2}}$ pushed us to opt for a double masking strategy, as exemplified with probes $\mathbf{P}_{3}$ and $\mathbf{P}_{4}$, which finally enable us to obtain much more stable boronate probes while keeping a good reactivity towards $\mathrm{H}_{2} \mathrm{O}_{2}$. With these doubly protected probes, detection of nanomolar concentrations of $\mathrm{H}_{2} \mathrm{O}_{2}$ in less than 10 min was achieved using a simple UV-visible absorbance readout, which in terms of analytical performance is competitive with the best fluorescent-based molecular probes currently available on the market for quantitative analysis of $\mathrm{H}_{2} \mathrm{O}_{2}$ by fluorescence. ${ }^{39-41}$ Finally, we will show that these excellent analytical performances could be obtained thanks to a comprehensive kinetic analysis of the complex cross-catalytic reaction network, thus providing an access to its quantitative prediction and rationalization. In particular, we were able to explain why the autocatalysis is still possible for doubly protected probes even in the absence of instability of the monoprotected intermediate.

\section{Results and discussion}

\section{Characterization of the single-protected probe $\boldsymbol{P}_{\mathbf{2}}$}

The ability to induce an autocatalytic reaction by mixing $\mathbf{P}_{2}, \mathrm{H}_{2} \mathrm{O}_{2}$ and a reducing agent has been investigated in a Tris buffer at $\mathrm{pH}$ 8.5. As a reductant, we used the same enzyme/reducing substrate as previously, i.e. the diaphorase/NADH couple. ${ }^{34}$ The diaphorase (DI) from Bacillus stearothermophilus (EC 1.6.99.-) is a thermostable flavin mononucleotide-dependent oxidoreductase that efficiently catalyzes the obligatory two-electron reduction of naphthoquinones into naphthohydroquinones in the $\mathrm{pH}$ range 7 to $10 . .^{42}$ It is also insensitive to $\mathrm{O}_{2}$ and its $\mathrm{NADH}$ substrate is quite stable in slightly basic aerated buffers ${ }^{43}$ (showing almost no cross-reactivity with dissolved dioxygen unless either $\mathrm{O}_{2}$ or $\mathrm{NADH}$ is activated ${ }^{44}$ ). The time course of the autocatalytic reaction was monitored by UVvisible spectrophotometry at $\lambda=340 \mathrm{~nm}$, a wavelength characteristic of the transformation of NADH into $\mathrm{NAD}^{+}$. A spectrophotometric microplate reader for high-throughput kinetic analysis was used to simultaneously monitor a set of different reaction mixtures $(200 \mu \mathrm{L}$ working solution per microwell). During the whole reaction, the microplate was left open to the atmosphere so as to operate under an approximately constant $\mathrm{O}_{2}$ concentration.

Kinetic traces recorded at different $\mathrm{H}_{2} \mathrm{O}_{2}$ concentrations are shown in Figure $1 \mathrm{~A}$. Their particularly steep sigmoidal shape is characteristic of an efficient autocatalytic process, for which the lag phase that precedes the exponential decrease of absorbance is a function of the triggering $\mathrm{H}_{2} \mathrm{O}_{2}$ concentration. Indeed, it is observed that the lower the starting $\mathrm{H}_{2} \mathrm{O}_{2}$ concentration, the longer the lag phase. It is also important to note the $\mathrm{S}$-shaped kinetic curve obtained in the absence of $\mathrm{H}_{2} \mathrm{O}_{2}$ (blank) attests to a nonspecific autocatalysis triggered by undesirable reactions (the latter initiating the production of either $\mathrm{H}_{2} \mathrm{O}_{2}$ or $\mathrm{Q} / \mathrm{QH}_{2}$ to eventually lead to the exponential and full consumption of NADH). The time-to-threshold values (i.e., $t_{1 / 2}$ defined here as the time required to reach $50 \%$ of the maximal absorbance change) extracted from these kinetic traces were used to plot, on a semi-logarithmic scale, the $\mathrm{H}_{2} \mathrm{O}_{2}$ calibration curve reported in Figure $1 \mathrm{~B}$. The fact that this calibration plot shows a quasilinear relationship between $t_{1 / 2}$ and $\log \left[\mathrm{H}_{2} \mathrm{O}_{2}\right]$ over nearly two order of magnitude (i.e., from 0.5 to $20 \mu \mathrm{M} \mathrm{H}_{2} \mathrm{O}_{2}$ ) confirms the autocatalytic nature of the reaction. The deviation from linearity observed at the lowest concentrations (with a tendency to merge progressively with the $t_{1 / 2}$ value determined in the absence of $\mathrm{H}_{2} \mathrm{O}_{2}$ ) illustrates the limitation imposed by the nonspecific response on the analytical performances, leading here to a LOD of
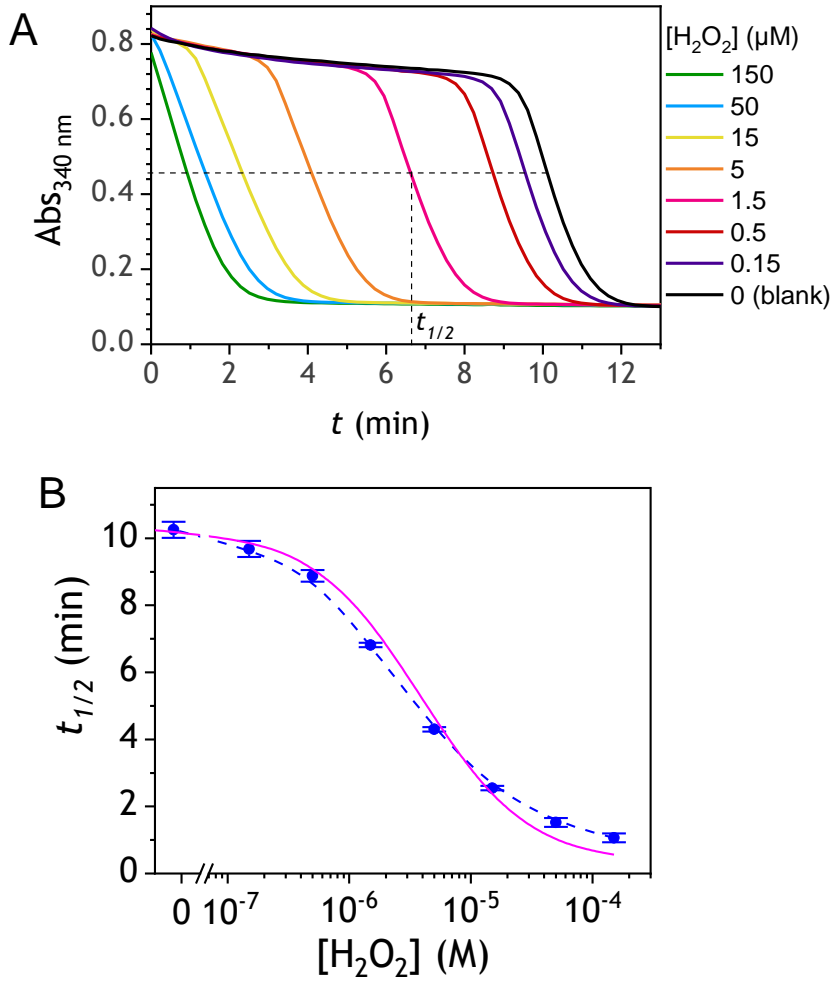

Figure 1. (A) UV-vis kinetic traces monitored at $340 \mathrm{~nm}$ in a 96-wells microtiter plate during the reaction generated from the mixing of different concentrations of $\mathrm{H}_{2} \mathrm{O}_{2}$ (see the caption on the graph) to solutions containing $50 \mu \mathrm{M} \mathrm{P} \mathbf{P}_{2}, 10 \mathrm{nM}$ DI and $250 \mu \mathrm{M}$ NADH. All experiments were conducted in an airsaturated Tris buffer (o.1 M, pH 8.5) containing $10 \mu \mathrm{M}$ EDTA. Each curve is the average of triplicate experiments. (B) Semilogarithmic calibration curve obtained from the threshold times $\left(t_{1 / 2}\right)$ recovered from the crossing of the horizontal dashed line in A to the kinetic traces $\left(t_{1 / 2}\right.$ is defined here as the time for which the absorbance is decreased approximatively half to the maximal absorbance change). Errors bars are standard deviations from triplicates. The magenta solid line is the theoretical plot obtained by simulation (see text and SI for details). 
only $0.2 \mu \mathrm{M} \mathrm{H}_{2} \mathrm{O}_{2}$, a value which is finally not much different from that we had previously obtained with the self-immolating probe $\mathbf{P}_{\mathbf{1}}$ under similar experimental conditions. ${ }^{34}$ Erreur ! Signet non défini. Nevertheless, the kinetics with $\mathbf{P}_{\mathbf{2}}$ are much faster than previously (by a factor 6), which is clearly an advantage to shorten the analysis time. The influence of $\mathrm{pH}$ was investigated by repeating the same experiments at two slightly less basic pHs (i.e., $\mathrm{pH} 8$ and $\mathrm{pH} 7 \cdot 5$ ). The resulting plots in Figure $\mathrm{S}_{1}$ show a shift in the kinetic traces to longer times along with a reduction in the slope of the sigmoid as the $\mathrm{pH}$ decreases, a behavior consistent with slower autoxidation and deprotection rates at lower $\mathrm{pHs} .{ }^{34}$ Unfortunately, no gain in LOD was obtained, suggesting a relatively similar $\mathrm{pH}$ dependence of both probe reactivity and stability. ${ }^{36}$

To understand why this new probe results in such a fast autocatalysis but also in this unexpectedly large nonspecific response, we have examined the fate of the probe alone in a Tris buffer ( $\mathrm{pH} \mathrm{8.5)}$ and its reactivity towards $\mathrm{H}_{2} \mathrm{O}_{2}$. The rather fast evolution of the UV-vis spectrum of a dilute solution of $\mathbf{P}_{\mathbf{2}}(50 \mu \mathrm{M})$ over 20 minutes (graph $\mathrm{A}$ in Figure $\mathrm{S}_{2}$ ) indicates the relatively poor stability of $\mathbf{P}_{\mathbf{2}}$ in the aqueous buffer. Moreover, the lack of isobestic point reveals the probe degradation into several byproducts. Their structural identity was determined from analyses by HPLC and comparison of the UV-visible spectra of degraded probe with reference spectra of different byproduct candidates (see Figures $\mathrm{S}_{2}, \mathrm{~S}_{3}, \mathrm{~S}_{4}$ and SI for details). Independently of the naphthol boronic acid derivative generated from fast hydrolysis of the pinacol group, two byproducts were found, i.e. the 1-naphthol and 1,4-NQ (according to HPLC analysis, in a 1:3 ratio in the fully degraded probe solution). The formation of 1-naphthol is the consequence of the slow first-order protodeboronation of $\mathbf{P}_{2}$, a well-established reaction for aromatic boronates in aqueous media, ${ }^{45,}{ }^{46}$ while the formation of $1,4-N Q$ was attributed to the slow oxidation of $\mathrm{C}$ - $\mathrm{B}$ bond by dissolved $\mathrm{O}_{2}$ (indeed, under argon, we observed that $\mathbf{P}_{2}$ and its hydrolyzed homologue only degrade into 1-naphthol - see Figure S3). Since the mechanism of this slow probe autoxidation is unknown, we have at first assumed it can be assimilated to a bimolecular reaction between $\mathbf{P}_{2}$ and $\mathrm{O}_{2}$, leading to $\mathrm{H}_{2} \mathrm{O}_{2}$ and 1,4$\mathrm{NQH}_{2}$. Even if slow, this probe autoxidation is clearly a major issue because, unlike protodeboronation which leads to the production of a dead-end product, it triggers the catalytic degradation of $\mathbf{P}_{2}$ via the production of $1,4-\mathrm{NQH}_{2}$ and regeneration of $\mathrm{H}_{2} \mathrm{O}_{2}$, which in turns further deprotects $\mathbf{P}_{2}$ until the latter is fully converted (Scheme 2). This undesirable reaction finally ends up in an important nonspecific response in the absence of $\mathrm{H}_{2} \mathrm{O}_{2}$, which is of course detrimental to the analytical performances. Owing to the pinacol hydrolysis occurring together with the other two nonspecific competitive reactions, it was tricky to access the individual kinetics of these reactions. For the sake of simplicity, we have assumed that the 3 rate constants characterizing the probe protodeboronation $\left(k_{p}\right)$, autoxidation $\left(k_{n}\right)$, and $\mathrm{H}_{2} \mathrm{O}_{2}$ deprotection $\left(k_{d}\right)$ are almost the same either
$\mathbf{P}_{2}$ is with or without its pinacol group. To determine these rates constants, we relied on a UV-vis absorbance measurement at $262 \mathrm{~nm}$, a wavelength selective of the 1,4-NQ.34 The kinetic traces in Figure $2 \mathrm{~A}$ and $\mathrm{S}_{5}$ were obtained either in the absence or presence of different $\mathrm{H}_{2} \mathrm{O}_{2}$ concentrations. Without $\mathrm{H}_{2} \mathrm{O}_{2}$, the change in absorbance is characterized by a small induction period followed by a nonlinear increase until it asymptotically reaches a constant value after more than $20 \mathrm{~min}$. This increase in absorbance indirectly measures the autoxidation rate of $\mathbf{P}_{\mathbf{2}}$ through the nonspecific catalytic formation of $1,4-\mathrm{NQ}$, from which we can estimate $k_{n}$. In the presence of $\mathrm{H}_{2} \mathrm{O}_{2}$, the increase in absorbance starts sooner (and without delay) and faster as the
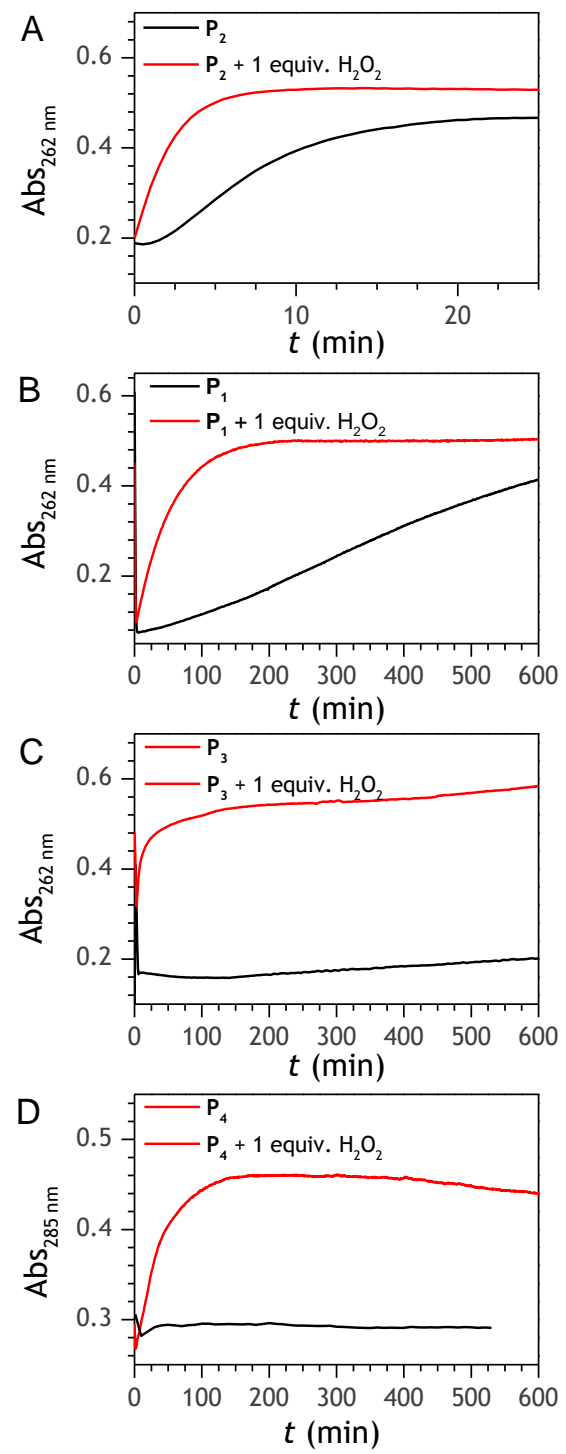

Figure 2. UV-vis kinetic traces recorded at 262 or $285 \mathrm{~nm}$ (wavelengths specific of 1,4-NQ) for solutions containing 50 $\mu \mathrm{M}$ of (A) $\mathbf{P}_{1}$, (B) $\mathbf{P}_{2}$, (C) $\mathbf{P}_{3}$, and (D) $\mathbf{P}_{4}$. The black lines correspond to the probe alone in solution, while the red lines correspond to a 1:1 mixture of probe and $\mathrm{H}_{2} \mathrm{O}_{2}$. All experiments were performed at $25^{\circ} \mathrm{C}$ in an air-saturated Tris buffer (o.1 M, $\mathrm{pH}$ 8.5) containing $10 \mu \mathrm{M}$ EDTA. 
Scheme 2. Reaction scheme associated to the instability of probe $P_{2}$ in aqueous solution.

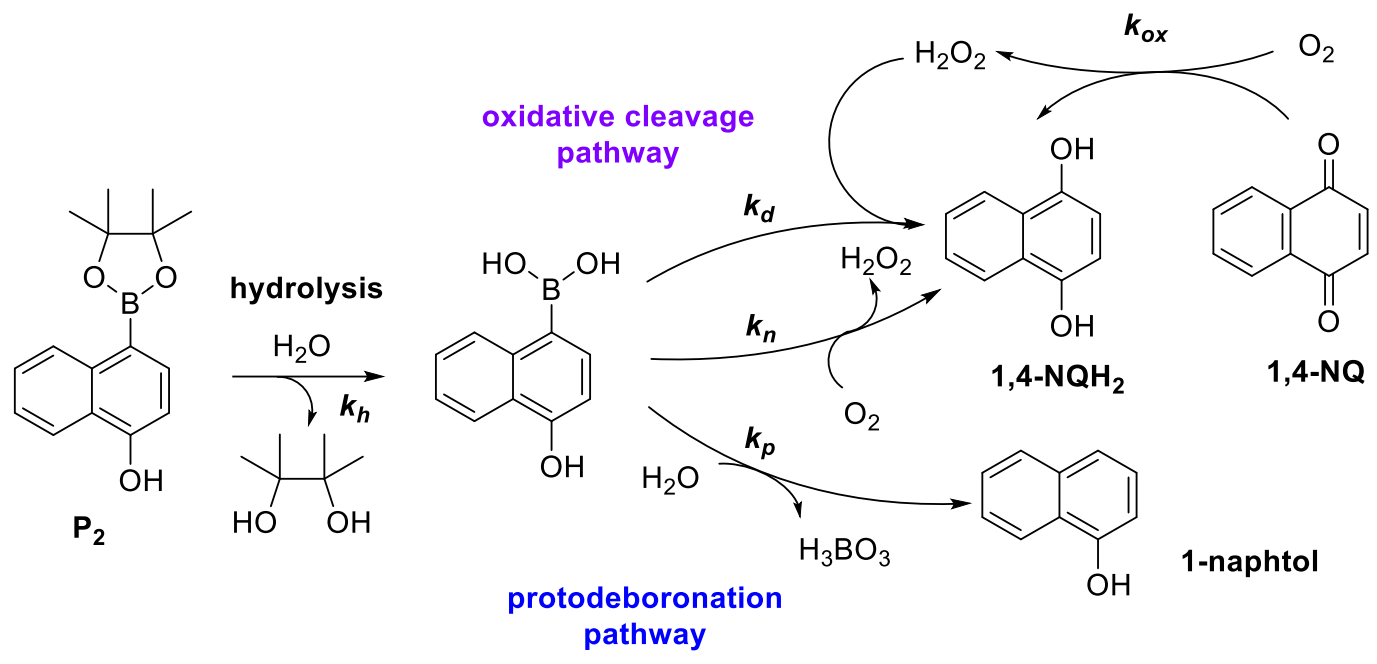

$\mathrm{H}_{2} \mathrm{O}_{2}$ concentration is higher (Figure $\mathrm{S}_{5}$ ). Furthermore, with the increase of $\left[\mathrm{H}_{2} \mathrm{O}_{2}\right]$, the maximum absorbance converges progressively towards a same maximal value, indicating complete probe transformation into 1,4-NQ. For the lowest and intermediate concentrations, the fact that the absorbance levels off at lower values suggests that a nonnegligible fraction of the probe converts into another compound, not detectable at this wavelength, which we attributed to 1-naphthol. The fast kinetics recorded in the presence of $\mathrm{H}_{2} \mathrm{O}_{2}$ demonstrate the fast $\mathrm{H}_{2} \mathrm{O}_{2}$-mediated deprotection of $\mathbf{P}_{2}$, clearly much faster than previously reported for the self-immolative probe $\mathbf{P}_{\mathbf{1}}$ (the kinetic traces of which are reminded in Figure $2 \mathrm{~B}$ for comparison). The trade-off is that the nonspecific self-deprotection of $\mathbf{P}_{\mathbf{2}}$ is also faster than $\mathbf{P}_{\mathbf{1}}$, therefore explaining the unsatisfactory detection limit of $\mathrm{H}_{2} \mathrm{O}_{2}$.

Assuming that the probe has similar reactivity, whether it is hydrolyzed or not, and the set of reaction in Scheme 2 is adequate to account for the different pathways of $\mathbf{P}_{\mathbf{2}}$ transformation, one can expect to reproduce by simulation the experimental curves in Figures $2 \mathrm{~A}$ and $\mathrm{S}_{5}$. For such purpose, we have considered for the $1,4-\mathrm{NQ}$ autoxidation the same global apparent bimolecular rate constant $\left(k_{o x}\right)$ than the one we had previously electrochemically determined (i.e., $3300 \mathrm{M}^{-1} \cdot \mathrm{s}^{-1}$ ), 34 a value high enough for the autoxidation of $1,4-\mathrm{NQH}_{2}$ to not be rate limiting. From iterative adjustment of the three other rate constants, i.e. $k_{d}, k_{n}$ and $k_{p}$, and assuming $\left[\mathrm{O}_{2}\right]$ constant, it was possible to reproduce quite well the experimental kinetic traces in Figure $\mathrm{S}_{5}$ using the following set of values: $k_{d}=300 \mathrm{M}^{-1} \cdot \mathrm{s}^{-1}, k_{n}=1.2 \mathrm{M}^{-1} \cdot \mathrm{s}$ ${ }^{1}$, and $k_{p}=4 \times 10^{-4} \mathrm{~s}^{-1}$. The extracted $k_{d}$ indicates a rather fast $\mathrm{H}_{2} \mathrm{O}_{2}$ deprotection, much faster than that previously determined for the arylboronate $\mathbf{P}_{\mathbf{1}}$ probe in a same Tris buffer $\left(\sim 5 \mathrm{M}^{-1} \cdot \mathrm{s}^{-1}\right) .34$ To our knowledge this is even the highest rate constant so far reported for an aromatic boronate compound (at neutral or slightly basic pHs, $\mathrm{H}_{2} \mathrm{O}_{2}$-mediated deprotection rate constants of arylboronates generally range from 0.07 to $\left.4 \mathrm{M}^{-1} \cdot \mathrm{s}^{-1} 45,47^{-51}\right)$. We attribute this to the presence of the hydroxy group in para position of the naphthylboronate, whose effect is probably to destabilize the C$\mathrm{B}$ bond by inductive and/or mesomeric effects. This is supported by the much lower $k_{d}$ value we separately determined for the 1-naphtylboronate in the same Tris buffer (i.e., $7 \cdot 5 \mathrm{M}^{-1} \cdot \mathrm{s}^{-1}$ ). The protodeboronation rate is also significantly higher (by ca. one order of magnitude) than previously reported for arylboronic acids, 45 a behavior we also attribute to the same electronic effects.

Investigation of the double-protected probe $\boldsymbol{P}_{3}$

To address the instability issue of the single-protected probe $\mathbf{P}_{2}$, we hypothesized that double substitution of the -OH groups of $1,4-\mathrm{NQH}_{2}$ by boronates could make the probe more stable. Therefore, the probe $\mathbf{P}_{3}$ was synthesized and then tested for its long-term stability in the Tris buffer. The data in Figure ${ }_{2} \mathrm{C}$ show the absorbance of a dilute $\mathbf{P}_{3}$ solution (recorded at $262 \mathrm{~nm}$, for an overview of the full spectrum see Figure S6), which after a rapid decrease during the first few minutes (ensuing from the hydrolysis of pinacols), stabilizes at $\sim 0.17$ over several hours. This result clearly demonstrates the high chemical stability of $\mathbf{P}_{3}$, which once hydrolyzed into diboronic acid does no longer decomposes into 1,4-NQ, therefore validating our initial hypothesis. This much higher chemical stability also correlates with a slower protodeboronation rate since the kinetic trace recorded at $290 \mathrm{~nm}$ (specific to the probe absorbance) is also very stable over several hours (see Figure $\mathrm{S}_{7}$ ). Adding one equivalent of $\mathrm{H}_{2} \mathrm{O}_{2}$ to the solution results in a fast absorbance increase at $262 \mathrm{~nm}$, rising to a maximal value in less than 5 min (red plot in Figure $2 \mathrm{C}$ ). It thus attests to an efficient $\mathrm{H}_{2} \mathrm{O}_{2}$-mediated deprotection of $\mathbf{P}_{3}$ into 1,4-NQ and so to a high responsiveness of the doubly protected probe to $\mathrm{H}_{2} \mathrm{O}_{2}$. In order to determine the deprotection rate of $\mathbf{P}_{3}$, we repeated the experiment at different $\mathrm{H}_{2} \mathrm{O}_{2}$ concentrations (Figure S8). As expected, the kinetics show a faster increase in absorbance as the $\mathrm{H}_{2} \mathrm{O}_{2}$ concentration is higher. However, unlike $\mathbf{P}_{\mathbf{2}}$ the maximal absorbance change scales with the stoichiometry of $\mathrm{H}_{2} \mathrm{O}_{2}$ (see SI 
and Figure S8 for details), which means that for each substoichiometric $\mathrm{H}_{2} \mathrm{O}_{2}$ concentration added to the solution of $\mathbf{P}_{3}$, only the corresponding substoichiometric equivalent in $[1,4-N Q]$ can be recovered. This distinct behavior of $\mathbf{P}_{3}$ can be explained from the need to provide two equivalents of $\mathrm{H}_{2} \mathrm{O}_{2}$ for a full deprotection of one equivalent, but also from the ability to regenerate an additional equivalent of $\mathrm{H}_{2} \mathrm{O}_{2}$ from each released molecule of $1,4-\mathrm{NQH}_{2}$. This also points out that the autoxidation of $1,4-\mathrm{NQH}_{2}$ is an efficient process for producing $\mathrm{H}_{2} \mathrm{O}_{2}$ at a yield close to $100 \%$ (a result which agrees with what was previously reported for the autoxidation of $1,4-\mathrm{NQ}^{52}$ ). The experimental kinetics reported in Figure S8 should therefore fulfil the mechanism presented by reactions $\mathrm{S}_{5}$ to $\mathrm{S}_{9}$ in $\mathrm{SI}$, wherein $\mathbf{P}_{3}$ is first converted into the monoprotected intermediate $\mathbf{P}_{2}$ and then into 1,4-NQ according to the same $\mathrm{H}_{2} \mathrm{O}_{2}$-mediated catalytic transformation described above for $\mathbf{P}_{2}$. This reaction scheme is therefore characterized by two deprotection rate constants, namely $k_{d, \mathrm{P}_{3}}$ and $k_{d, \mathrm{P}_{2}}$. The value of $k_{d, \mathrm{P}_{2}}$ can be considered the same than the one determined above from $\mathbf{P}_{2}$, a consideration which remains also valid for all the other rate constants, the only new rate being $k_{d, \mathrm{P}_{3}}$. We have thus numerically simulated the set of reactions $\mathrm{S}_{5}$ to S9 using the value $k_{d, \mathrm{P}_{2}}, k_{o x}, k_{n}$ and $k_{p}$ previously recovered from $\mathbf{P}_{2}$ and iteratively adjusted the rate constant $k_{d, \mathrm{P}_{3}}$. The best fits of the simulated curves to the experimental data are shown in Figure S8 (dashed plots in graph D) using $k_{d, \mathrm{P}_{3}}=35 \mathrm{M}^{-1} \cdot \mathrm{s}^{-1}$. Despite all of our attempts, it was not possible to perfectly fit the data, the simulated plots showing a systematic slower increase of $1,4-N Q$ with time whatever $k_{d, \mathrm{P}_{3}}$. In fact, much better fits were obtained by artificially considering the second deprotection rate fast enough (e.g., $\left.k_{d, \mathrm{P}_{2}}>3000 \mathrm{M}^{-1} \cdot \mathrm{s}^{-1}\right)$ to no longer interfere on the kinetics (solid plots in graph D of Figure S8). This apparent inconsistency suggests that in parallel to the $\mathrm{H}_{2} \mathrm{O}_{2}$ deprotection of the intermediate $\mathbf{P}_{2}$, additional fast reactions contribute to an accelerated deprotection of $\mathbf{P}_{\mathbf{2}}$. We emitted the hypothesis that it could be due to the autoxidation of $\mathbf{P}_{2}$ (reaction S8), which instead of a two-electron transfer reaction, as we have at first assumed, occurs through a one-electron transfer between $\mathrm{O}_{2}$ and $\mathbf{P}_{2}$ with the concomitant production of radical species (notably $\mathrm{O}_{2}{ }^{\cdot}$ , which by dismutation can produce $\mathrm{H}_{2} \mathrm{O}_{2}$ for additional deprotection of both $\mathbf{P}_{3}$ and intermediate $\mathbf{P}_{2}$ ). One can then conceive that these radical species can either directly or indirectly further contribute to the accelerated deprotection of the intermediate $\mathbf{P}_{\mathbf{2}}$. We will come back later on this specific point. It is to note that the value of $k_{d, \mathrm{P}_{3}}=35$ $\mathrm{M}^{-1} \cdot \mathrm{s}^{-1}$ recovered from the best fits, even if lower than $k_{d, \mathrm{P}_{2}}$ , remains high and attractive for an efficient autocatalysis.

Autocatalysis with $\boldsymbol{P}_{3}$
The autocatalysis with $\mathrm{P}_{3}$ was performed under the same conditions than $\mathbf{P}_{2}$. The resulting kinetics curves (see Figure $3 \mathrm{~A}$ ) characterizing the NADH consumption as a function of time, show, similarly to $\mathbf{P}_{2}$, well-defined S-shaped kinetics traces with longer lags as the triggering $\mathrm{H}_{2} \mathrm{O}_{2}$ concentration is lower. However, a main difference with $\mathbf{P}_{\mathbf{2}}$ is the much slower kinetics, requiring for the lowest concentrations more than 5 hrs to reach $t_{1 / 2}$, while it takes less than 10 min with $\mathbf{P}_{2}$. Such a huge difference can be understood from the slower deprotection rate of $\mathbf{P}_{3}$ with respect to $\mathbf{P}_{2}$, as well as from the need of two equivalents of $\mathrm{H}_{2} \mathrm{O}_{2}$ to fully deprotect each $\mathbf{P}_{3}$ molecule. The analytical performances
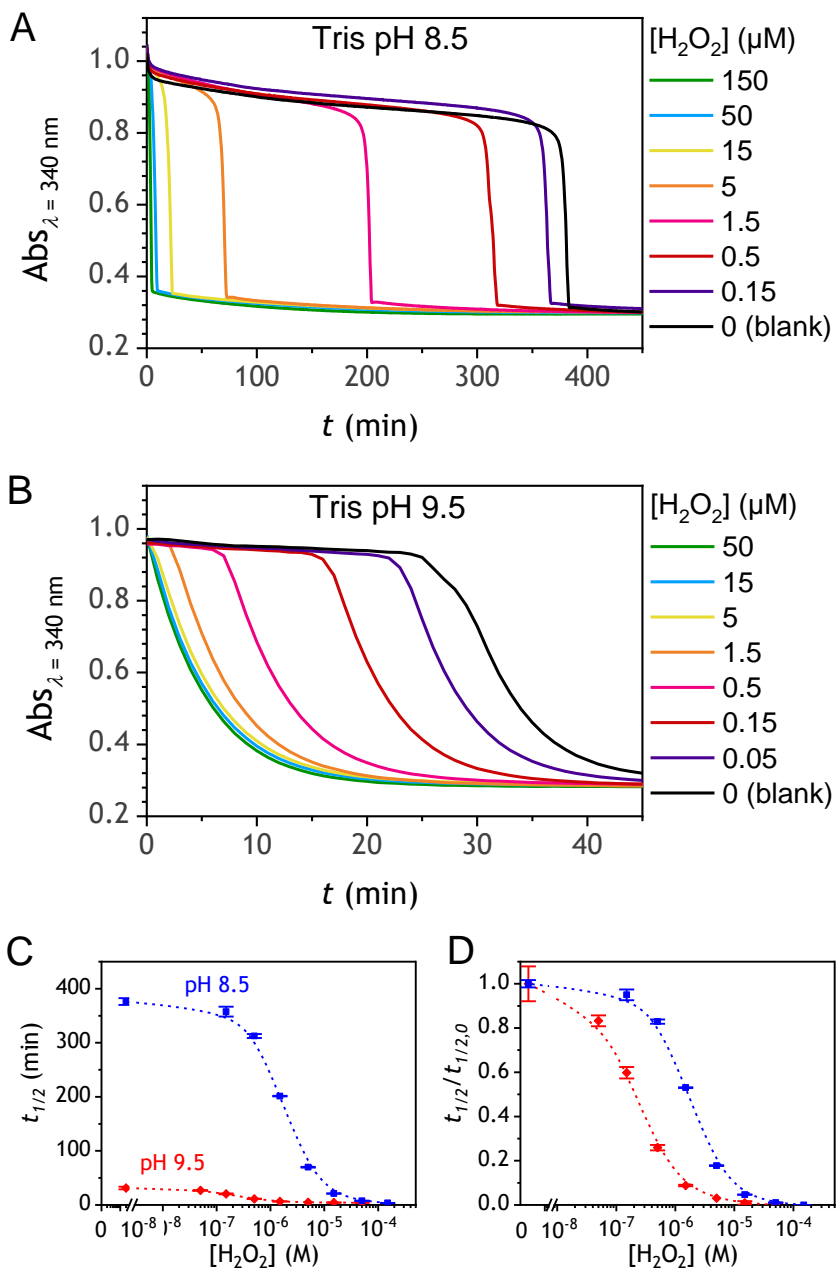

Figure 3. (A, B) UV-vis kinetic traces monitored at $340 \mathrm{~nm}$ immediately after the injection of different concentrations of $\mathrm{H}_{2} \mathrm{O}_{2}$ (see the caption on the graph) to solutions containing 50 $\mu \mathrm{M} \mathbf{P}_{3}, 10 \mathrm{nM}$ DI and $250 \mu \mathrm{M}$ NADH. The experiments were conducted in an air-saturated Tris buffer (o.1 M) of (A) pH 8.5 or $(\mathrm{B}) \mathrm{pH}$ 9.5. Each curve is the average of triplicate experiments. (C) Semi-logarithmic calibration curves obtained from the plot of $t_{1 / 2}$ (recovered from the top and middle kinetic traces) as a function of $\log \left[\mathrm{H}_{2} \mathrm{O}_{2}\right]$. Red dots: $\mathrm{pH}$ 9.5. Blue dots: $\mathrm{pH}$ 8.5. Errors bars are standard deviations from triplicates. (D) Same graphs than in C but normalized to the $t_{1 / 2,0}$ recovered from the blank. 
grasped from the semi-logarithmic calibration plot in Figure ${ }_{3} \mathrm{C}$ indicate a dynamic concentration range over more than two decades (i.e., from o.1 to $20 \mu \mathrm{M} \mathrm{H}_{2} \mathrm{O}_{2}$ ) and a LOD of $0.2 \mu \mathrm{M} \mathrm{H}_{2} \mathrm{O}_{2}$. These analytical performances are not as good as expected, leading to a LOD similar to that of $\mathbf{P}_{2}$, which is surprising if we consider the much higher chemical stability of $\mathbf{P}_{3}$. In order to improve the reaction rate and at the same time the analytical performances, we reproduced the autocatalysis at a slightly more basic $\mathrm{pH}(\mathrm{pH}$ 9.5). The resulting autocatalytic responses in Figure ${ }_{3} \mathrm{~B}$ show a clear kinetic gain, reducing the $t_{1 / 2}$ values to a time window $<40 \mathrm{~min}$. Compared to the results obtained at $\mathrm{pH}$ 8.5, this corresponds to an analysis time improvement by a factor 10. Moreover, the extracted $\mathrm{H}_{2} \mathrm{O}_{2}$ calibration curve (Figure ${ }_{3} \mathrm{C}$ ) demonstrates a better LOD of $\sim 20 \mathrm{nM}$, which is again a 10-fold improvement compared to $\mathrm{pH} 8.5$ (Figure $3 \mathrm{D})$. Clearly, the $\mathrm{pH}$ has a strong impact on the analytical performances of the system, since by changing the $\mathrm{pH}$ of one unit, both LOD and analysis time are improved 1o-fold. All these results led us to investigate what could lead to such a drastic $\mathrm{pH}$ effect, but also how to interpret the large rate difference between $\mathbf{P}_{3}$ and $\mathbf{P}_{2}$. To this end, we examined the possibility to simulate the autocatalytic kinetic traces on the basis of a reasonable set of reactions for describing the cross coupling between the $\mathrm{H}_{2} \mathrm{O}_{2}$-mediated catalytic deprotection of $\mathbf{P}_{3}$ (Loop 1) and the enzyme-assisted redox cycling (Loop 2) (see thereafter).

Mechanistic and kinetics analysis of the autocatalytic process

The catalyzed reduction of quinones by DI is a well-established "ping-pong" mechanism, ${ }^{42}$ which under turnover conditions can be defined by two half enzyme reactions (and three rate constants), i.e. (i) a two-step MichaelisMenten type reaction between NADH and the oxidized form of the enzyme (characterized thus by a MichaelisMenten constant $K_{\mathrm{M}}$ and a catalytic turnover rate constant $k_{\mathrm{c}}$ ) and (ii) a bimolecular reaction characterizing the obligatory two-electron transfer reduction of quinones (here the $1,4-\mathrm{NQ}$ ) by the reduced form of the enzyme (governed thus by a second-order rate constant $k_{\text {red }}$ ). The coupling of this enzymatic "ping-pong" mechanism to the $\mathrm{H}_{2} \mathrm{O}_{2}$-mediated deprotection of $\mathbf{P}_{3}$ leads to the autocatalytic set of reactions S1o to S16 in SI. To simulate this mechanism, we first used for Loop 2 the values of $K_{\mathrm{M}}, k_{c}$ and $k_{\text {red }}$ (i.e., 6o $\mu \mathrm{M}, 1200 \mathrm{~s}^{-1}, 1.5 \times 10^{9} \mathrm{M}^{-1} \mathrm{~s}^{-1}$, respectively) previously determined by us for an analogue of the $1,4-\mathrm{NQ}$, i.e. the menadione, ${ }^{42}$ while the other rate constants associated to Loop 1 were the same as those above determined (for clarity, the list of rate constants are gathered in the entry 1 of Table 1 ). The simulated time course of NADH consumption at different $\mathrm{H}_{2} \mathrm{O}_{2}$ concentrations are shown in Figure $\mathrm{S}_{9}$. Their sigmoidal shapes are, as expected, in agreement with an autocatalytic reaction and also similar with those obtained experimentally (Figure $3 \mathrm{~A}$ ). However, a main issue is that their $t_{1 / 2}$ do not match those obtained experimentally, differing by a factor as high as 28 . This result indicates that something is missing in the proposed mechanism. We figured out that it might be due to our oversimplified description of the autoxidation of $1,4-\mathrm{NQ}$. It is well established that the autoxidation mechanism of hydroquinones is more complex than a simple bimolecular reaction. It is indeed an autoinductive radical chain mechanism, in which the semiquinone $\left(\mathrm{Q}^{-}\right)$and anion superoxide are involved as chain propagating species as follow: :2-54 $^{2}$

$$
\begin{aligned}
& \mathrm{QH}_{2}+\mathrm{O}_{2} \stackrel{k_{0}}{\longrightarrow} \mathrm{Q}^{\cdot-}+\mathrm{O}_{2}^{\cdot-}+2 \mathrm{H}^{+} \\
& \mathrm{QH}_{2}+\mathrm{Q} \underset{k_{d s,-1}}{\stackrel{k_{c o, 1}}{\rightleftarrows}} 2 \mathrm{Q}^{\cdot-}+2 \mathrm{H}^{+} \\
& \mathrm{Q}^{\cdot-}+\mathrm{O}_{2} \underset{k_{-2}}{\stackrel{k_{2}}{\rightleftarrows}} \mathrm{Q}+\mathrm{O}_{2}^{\cdot-} \\
& \mathrm{QH}_{2}+\mathrm{O}_{2}^{\cdot-} \stackrel{k_{3}}{\longrightarrow} \mathrm{Q}^{\cdot-}+\mathrm{H}_{2} \mathrm{O}_{2} \\
& 2 \mathrm{O}_{2}^{\cdot-} \stackrel{k_{\text {dis: }}}{\longrightarrow} \mathrm{O}_{2}+\mathrm{H}_{2} \mathrm{O}_{2} \\
& \mathrm{Q}^{0^{-}}+\mathrm{O}_{2}^{\bullet^{--}}+2 \mathrm{H}^{+} \stackrel{k_{5}}{\longrightarrow} \mathrm{Q}+\mathrm{H}_{2} \mathrm{O}_{2}
\end{aligned}
$$

This chain reaction is initiated by reaction o, which is assumed to be quite slow under near-neutral conditions because spin-restricted. ${ }^{55}$ However, once a small amount of $\mathrm{Q}^{-}$is produced, it is able to trigger a cascade of faster reactions, notably reactions 1,2 and 5 (forming a set of propagation reactions), which results in an autoinductive acceleration of the oxidation of 1,4-NQ by $\mathrm{O}_{2}$. Through this reaction network, $\mathrm{QH}_{2}$ and $\mathrm{O}_{2}$ are consumed by reactions 1, 3 and 2, respectively, while $\mathrm{Q}$ is produced in oxidation reactions 2 and 5. This reaction scheme predicts a 1:1 stoichiometry for the autoxidation of $\mathrm{QH}_{2}$, leading thus to a 1:1 production of $Q$ and $\mathrm{H}_{2} \mathrm{O}_{2}$. The rate acceleration of this mechanism originates from the product $\mathrm{Q}$, which is the catalyst of its own formation through the comproportionation reaction 1 . The majority of these reactions are $\mathrm{pH}$-dependent but, for sake of simplicity, the rate constants used in this work are those at an effective $\mathrm{pH}$ (here $\mathrm{pH}$ 8.5). The rate constant values we have used are gathered in Table 1. Most of them were retrieved from the literature. A few were corrected for $\mathrm{pH}$, while others were adjusted to fit the experimental data (see SI for a detailed description of the rate constant selection).

Incorporating the above autoxidation mechanism of $1,4^{-}$ $\mathrm{NQH}_{2}$ to the coupled enzymatic reduction of $1,4-\mathrm{NQ}$ and $\mathrm{H}_{2} \mathrm{O}_{2}$-mediated deprotection of $\mathbf{P}_{\mathbf{3}}$ leads to the autocatalytic set depicted in Scheme 3. Furthermore, since $\mathbf{P}_{3}$ is very stable and therefore unlikely to be the source of the nonspecific triggering of autocatalysis, the alternative is to consider it originates from the slow autoxidation of NADH (characterized by the rate $k_{n s}$ ). In our model, this nonspecific reaction was assumed to be a one-electron transfer process, leading to the release of $\mathrm{O}_{2}^{-{ }^{-}}$and NAD' (defined as $\mathrm{S}^{-}$in Scheme 3). Moreover, as we will see later, this reaction is apparently catalyzed by DI (NADPH oxidase-like activity), which leads to a dependence of the nonspecific response on the enzyme concentration. Since there is no 
available information on the autoxidation kinetics of $\mathrm{NADH}$ in the literature (either it is catalyzed or not by DI), the value of $k_{n s}$ in our simulations was simply adjusted so that the simulated nonspecific autocatalytic response aligns with the experimental one. In this model, we also substituted the two-electron autoxidation of the intermediate $\mathbf{P}_{\mathbf{2}}$ by a one-electron transfer reaction so as to better reflect the radical chain reaction we suspect coupled to this reaction. The S-shaped kinetic curves numerically calculated using the model in Scheme 3 and the associated rate constants gathered in entry 2 of Table 1 are shown in Figure S9, while the theoretical $\mathrm{H}_{2} \mathrm{O}_{2}$ calibration curve derived from these plots is given in Figure 4 (green line). Compared to the previous simulations involving just a simple second order reaction between $1,4-\mathrm{NQH}_{2}$ and $\mathrm{O}_{2}$, the new computed kinetics traces are now much closer to the experimental ones, a results which highlights the importance to consider the overall complexity of $1,4-\mathrm{NQH}_{2}$ autoxidation.

Scheme 3. Overall reactions involve in the autocatalytic deprotection of $\mathrm{P}_{3}$ in the presence of the reducing NADH/DI couple and $\mathrm{H}_{2} \mathrm{O}_{2}$. The enzyme substrate $\mathrm{NADH}$ and product $\mathrm{NAD}^{+}$are here symbolized by $\mathrm{S}$ and $R$, and the different states of the diaphorase enzyme by $E_{o}$ (oxidized), $E_{r}$ (reduced) and ES (enzyme/substrate precursor complex).

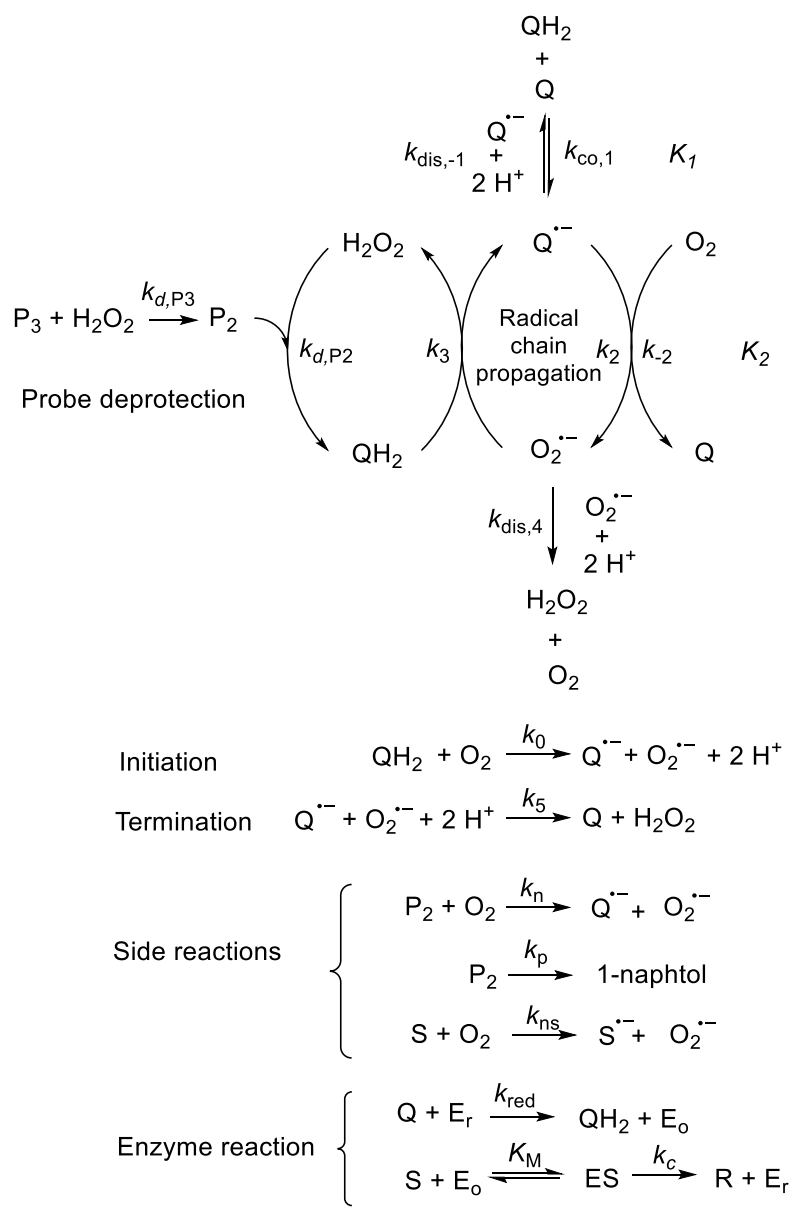

Interestingly, the discrepancy we observe between simulations and experiments is clearly on the side of the lowest $\mathrm{H}_{2} \mathrm{O}_{2}$ concentrations (Figure 4 ), since at the highest concentrations the theoretical plot fits the experimental data almost perfectly. We suspected that this could be related to an inhibition of the autoxidation of $\mathbf{P}_{2}$ intermediate induced by the presence of the reducing enzyme/NADH substrate. As we have already mentioned, the autoxidation reaction mechanism of $\mathbf{P}_{\mathbf{2}}$ could well be of a radical nature accompanied by a chain process. If we assume that this is the case, then one can consider that such a radical chain reaction is promoted under oxidizing conditions and inhibited under reducing conditions. To evaluate such an effect on the autocatalysis via simulation, we disabled in our model the autoxidation reaction of the monoboronic intermediate by setting its $k_{n}$ value to zero. Thanks to this, we were able to obtain the magenta simulated plot in Figure 4, which now predicts the experimental data much more closely. This finding tends to support the hypothesis that the slow self-deprotection of $\mathbf{P}_{2}$ by $\mathrm{O}_{2}$ occurs through a one-electron transfer reaction with concomitant formation of radical species (possibly $\mathrm{O}_{2}^{-{ }^{-}}$and a naphthoxyl radical), which are then inhibited or scavenged under the reducing conditions (NADH has been shown to efficiently scavenge either $\mathrm{HO}_{2}{ }^{\cdot 56}$ or phenoxyl radicals ${ }^{57}$ by one-electron reduction). This also demonstrates that the double masking does not prevent the autocatalytic reaction from working, even when the instability of the monoprotected intermediate is switched-off (i.e. when $k_{n}=0$ ). Indeed, when $k_{n}=0$, the autocatalysis still occurs with a reasonable efficiency (magenta curves in Figure 4) because, statistically, the probability of having a double-deprotection of the probe is not null, even under the substoichiometric $\mathrm{H}_{2} \mathrm{O}_{2}$ conditions used here. In fact, this probability depends directly on the ratio between the first and the second deprotection rate constants (i.e., $k_{d, \mathrm{P}_{3}}$ and $k_{d, \mathrm{P}_{2}}$ ), which is more favorable when the first one is slower than the second, as it is the case here (i.e., $35 v$ s. $300 \mathrm{M}^{-1} \cdot \mathrm{s}^{-1}$, respectively).

As the magenta simulated plot in Figure 4 still does not completely superimpose to the data, we considered that it could be due to the uncertainty in the selected values of $k_{3}$ and $k_{\text {red }}$ (the latter being moreover recovered from a previous enzymatic study performed with menadione but not with the 1,4-NQ). To experimentally access the value of these two rate constants, we have performed redox cycling experiments (Loop 2) by mixing a fixed concentration of DI (10 $\mathrm{nM})$ and $\mathrm{NADH}(250 \mu \mathrm{M})$ with different concentrations of $1,4-\mathrm{NQ}$ and then monitoring the NADH absorbance decay (see graph A in Figure Sio). From the results reported in Figure Sio and analyzed in details in SI, we were able to estimate the values of $k_{3}=(5-8) \times 10^{4} \mathrm{M}^{-1} \cdot \mathrm{s}^{-1}$ and $k_{\text {red }}=(1.5$ $-0.8) \times 10^{8} \mathrm{M}^{-1} \cdot \mathrm{s}^{-1}$ with a reasonably good degree of confidence. We thus considered these new values in our simulations and we succeeded to obtain an almost perfect agreement between the simulated calibration plot and the 


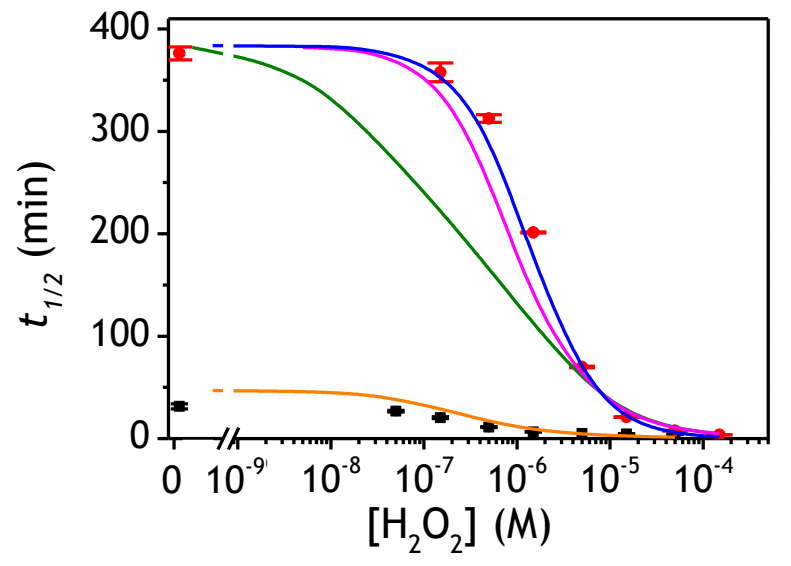

Figure 4. Plots of the experimental and theoretical semi-logarithmic $\mathrm{H}_{2} \mathrm{O}_{2}$ calibration curves. The red and black dots are the experimental data recovered for $\mathbf{P}_{3}$ at $\mathrm{pH} 8.5$ and 9.5 respectively (same data than in Figure ${ }_{3} \mathrm{C}$ and $\mathrm{D}$ ), while the solid lines are the theoretical plots predicted from numerical simulations of the autocatalytic set presented in Scheme 3. The parameters used for each simulated plots are those reported in Table 1 according to the following code color: (green) entry 2 , (magenta) entry 3, (bleu) entry 4, and (orange) entry 5.

experimental data using $k_{\text {red }}=10^{8} \mathrm{M}^{-1} \cdot \mathrm{s}^{-1}$ and $k_{3}=5 \times 10^{4} \mathrm{M}^{-}$ ${ }^{1} \cdot \mathrm{s}^{-1}$ (blue curve in Figure 4 ). This latter result definitely validates the model in Scheme 3 as well as the selected set of rate constants. With this tool in hand, it was next interesting to go back to the data with $\mathbf{P}_{\mathbf{2}}$ in order to see to what extent the autocatalytic kinetic traces in Figure 1 can be predicted by the model in Scheme 3 and the associated rate constants (excluding of course from the model the first deprotection which does not take place with $\mathbf{P}_{\mathbf{2}}$ ). The bestsimulated curves are reported in Figure $S_{12}$ using the rate constants gathered in the entry 6 of Table 1 . To obtain such a good fit, it was necessary to adjust the rate constant $k_{n}$ to a non-null value, which is not surprising as for simulating the nonspecific autocatalytic response of $\mathbf{P}_{2}$, it is now necessary to consider the probe autoxidation as the main source of nonspecificity (thus controlled by $k_{n}$ ) and no longer the NADH autoxidation $\left(k_{n s}\right)$. A $k_{n}$ of $0.25 \mathrm{M}^{-1} \cdot \mathrm{s}^{-1}$ was thus set from the alignment of the simulated nonspecific response to the experimental one. This value is significantly lower than the value of $1.2 \mathrm{M}^{-1} \cdot \mathrm{s}^{-1}$ previously derived from the $\mathrm{H}_{2} \mathrm{O}_{2}$-mediated deprotection of $\mathbf{P}_{2}$, therefore confirming that there is an inhibition of the autoxidation of $\mathbf{P}_{2}$ under the reducing conditions of autocatalysis. The theoretical $\mathrm{H}_{2} \mathrm{O}_{2}$ calibration plot that was finally extracted from the simulated kinetic traces fits nicely to the experimental calibration plot in Figure $1 \mathrm{~B}$. This is a strong plea for the validity of the proposed model. It also demonstrates the excellent self-consistency of our results. Armed with this knowledge, it was next interesting to identify the keys rate constants controlling the rate of autocatalysis but also to determine what limits the analytical performances of the method.
Rationalization of the effect of $\mathrm{pH}$ on the kinetics and LOD

To understand the influence of the $\mathrm{pH}$ on the rate of autocatalysis (as illustrated in Figure 3), one has to start by identifying the rate constants that a priori are the most strongly $\mathrm{pH}$ dependent. On account of the two $\mathrm{p} K_{a} \mathrm{~s}$ of the $1,4-\mathrm{NQH}_{2}\left(\mathrm{p} K_{\mathrm{a}, 1}=9.48\right.$ and $\mathrm{p} K_{\mathrm{a}, 2}=11.02$, see SI $)$, the rate constants $k_{0}, k_{\mathrm{co}, 1}$ and $k_{3}$ are clearly much $\mathrm{pH}$ sensitive than the other rate constants of the model (since this is $\mathrm{HO}_{2}{ }^{-}$the reactive species involved in the oxidative cleavage of boronates,,$^{8}$ the constants $k_{d, \mathrm{P}_{2}}$ or $k_{d, \mathrm{P}_{3}}$ are also $\mathrm{pH}$ dependent but to a lesser extent than $k_{o}, k_{\mathrm{co}, 1}$ and $\left.k_{3}\right)$. Therefore, if we consider $k_{\mathrm{co}, 1}$ and $k_{3}\left(k_{o}\right.$ is not taken into account for the same reason as previously) are mainly responsible for the $\mathrm{pH}$ dependency of the autocatalysis rate, this should be verifiable through the simulation of the $\mathrm{H}_{2} \mathrm{O}_{2}$ calibration curve. From the two $\mathrm{p} K_{a} \mathrm{~s}$ of the $1,4-\mathrm{NQH}_{2}$, we can calculate the values of $k_{c o, 1}$ and $k_{3}$ at $\mathrm{pH} 9 \cdot 5$, which are $6.6 \times 10^{6} \mathrm{M}^{-1} \cdot \mathrm{s}$ ${ }^{1}$ and $4.7 \times 10^{6} \mathrm{M}^{-1} \cdot \mathrm{s}^{-1}$, respectively (see SI). The theoretical $\mathrm{H}_{2} \mathrm{O}_{2}$ calibration curve (orange line in Figure 4 ) determined using these new values of $k_{c o, 1}$ and $k_{3}$ (while keeping the other rate constants the same as previously at $\mathrm{pH} 8.5$ ) finally agrees quite well with the experimental plot at $\mathrm{pH}$ 9.5 (black squares in Figure 4). This result confirms the key role played by both $k_{c o, 1}$ and $k_{3}$ in the $\mathrm{pH}$-rate dependence of the autocatalysis. A better fit to the data would certainly be obtained by increasing slightly the two deprotection rate constants $k_{d, \mathrm{P}_{2}}$ and $k_{d, \mathrm{P}_{3}}$, which, as mentioned above, are also expected to rise with the $\mathrm{pH}$. This kinetic analysis also explains the better detection limit obtained by increasing the $\mathrm{pH}$ (Figure $3 \mathrm{D}$ ). Indeed, if we admit that the NADH autoxidation $\left(k_{n s}\right)$ (which determines the nonspecific response of the system and thus the LOD) varies less rapidly with the $\mathrm{pH}$ than the overall rate of autocatalysis (varying predominantly with the $\mathrm{pH}$ through $k_{\mathrm{co}, 1}$ and $k_{3}$ ), then we can understand why the LOD is 10-fold improved when the $\mathrm{pH}$ is increased from 8.5 to 9.5.

\section{Further improving the analytical performances}

To determine whether the analytical performance could be further improved and/or optimized, we performed the autocatalysis reaction in buffers of different nature adjusted at different pHs (it is to note that DI from the Bacillus Stearothermophilus has a bell-shaped activity in the $\mathrm{pH}$ range 6.5 to 10.5 , with an optimum at $\mathrm{pH} 8.0^{59}$ ). Among the different organic and inorganic buffers investigated (see SI and Figures $\mathrm{S}_{13}$ to $\mathrm{S}_{20}$ ), the glycine buffer at $\mathrm{pH}$ 10 was found superior. The analytical performances obtained with this buffer were indeed clearly improved (Figure S18), leading to a dynamic concentration range over nearly 3 decades (i.e., from ca. o.o1 to $10 \mu \mathrm{M} \mathrm{H}_{2} \mathrm{O}_{2}$ ) and a LOD as low as $5 \mathrm{nM}$ $\mathrm{H}_{2} \mathrm{O}_{2}$ in less than 10 min reaction time. This is a very appealing performance since in terms of LOD, it is competitive with the best $\mathrm{H}_{2} \mathrm{O}_{2}$ detection kits currently commercially available (a LOD of $10 \mathrm{nM} \mathrm{H}_{2} \mathrm{O}_{2}$ within 30 min incubation time is reported for the popular fluorescent-based 
Amplex red ${ }^{\circledast}$ hydrogen peroxide/peroxidase assay ${ }^{41}$ ). Another buffer particularly attractive is the CAPS buffer at $\mathrm{pH}$ 9.5 (Figure S2O). In spite of a slightly longer reaction time, the data indicates the possibility to reach the nanomolar $\mathrm{H}_{2} \mathrm{O}_{2}$ concentration range in less than $50 \mathrm{~min}$. It is to note that with some buffers the autocatalysis was found to be inefficient in producing well-defined or discernible autocatalytic responses. This was especially true with the borate buffer (Figure $\mathrm{S}_{17}$ ). It was also observed in some buffers an anomalous shift of the nonspecific blank response to shorter times compared to the responses recorded at the lowest $\mathrm{H}_{2} \mathrm{O}_{2}$ concentrations. All these behaviors suggest that the buffer plays an active role in the autocatalysis that we have not yet been able to elucidate.

Another parameter we examined to improve the analytical performances was the concentration of DI. As shown in Figure 5, the concentration of the reducing enzyme was found to have a considerable impact on the nonspecific autocatalytic response, dramatically accelerating the nonspecific $t_{1 / 2}$ value with the rise of DI concentration, an effect which suggests that the enzyme slowly catalyzes the reaction between NADH and $\mathrm{O}_{2}$ to produce $\mathrm{O}_{2}{ }^{-}$or $\mathrm{H}_{2} \mathrm{O}_{2}$. These results echo the residual NADH oxidase activity $(<0.01 \%)$ reported by the enzyme supplier and which would
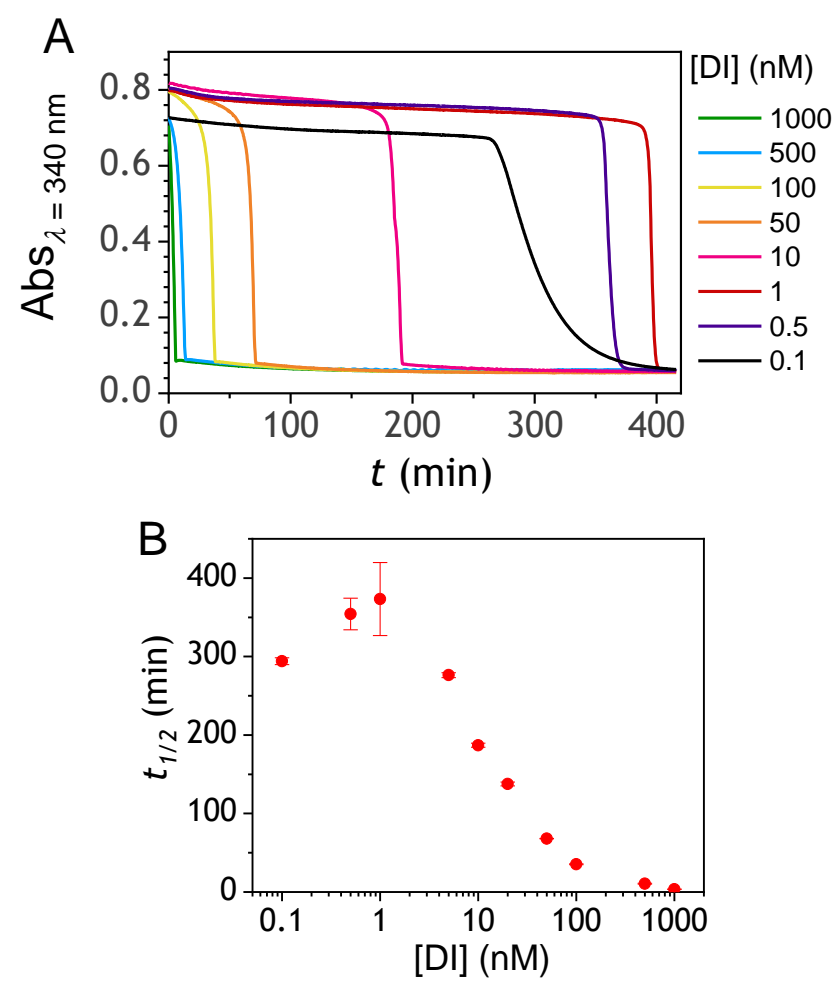

Figure 5. (Left) Influence of the reducing enzyme concentration on the nonspecific autocatalytic response. The kinetic traces at $340 \mathrm{~nm}$ were recorded immediately after $50 \mu \mathrm{M} \mathbf{P}_{3}$ was mixed with $250 \mu \mathrm{M}$ NADH and different concentrations of DI (see the legend on the graph) in an air-saturated Tris buffer (o.1 M) at pH 8.5. (Right) Semi-logarithmic curve showing the influence of the DI concentration on the $t_{1 / 2}$ values recovered from the nonspecific autocatalytic responses. be related to the presence of traces of NADH oxidase in the purified diaphorase samples. However, another possibility that cannot be ruled out is that DI has an intrinsic residual NADH oxidase activity. Indeed, one can assume that the reaction with $\mathrm{O}_{2}$ is not necessarily inexistent even though this flavoenzyme is known to not accept $\mathrm{O}_{2}$ as an electron acceptor. By gradually decreasing the concentration of DI, we can see in Figure 5 that the value of $t_{1 / 2}$ increases until it reaches a maximum before slightly decreasing at the lowest concentration (graph B in Figure 5). This indicates that by lowering the concentration of DI, the nonspecific contribution associated to the residual NADH oxidase activity is reduced. This can therefore be exploited to improve the specific $v s$. nonspecific response, especially if the enzyme reduction rate of the naphthoquinone in Loop 2 continues to be not rate limiting. This latter attribute can be easily verified from the $\mathrm{S}$-shape of the autocatalytic response. Indeed, if the kinetic profiles are similar regardless of the enzyme concentration, it suggests that the naphthoquinone reduction is not rate limiting. On the other hand, when the rate of the naphthoquinone reduction becomes too slow, as illustrated for the lowest enzyme concentration (i.e. o.1 $\mathrm{nM}$ in Figure 5), the kinetic trace presents a more spreadout autocatalytic response, signing thus the slowing down of Loop 2. The kinetic gain also observed at the lowest enzyme concentrations can be explained from the redox cycling (Loop 2) in Scheme 3, which tends to be inhibited at high DI concentration by forcing the naphthoquinone to be rapidly doubly reduced, thus bypassing the formation of semi-naphthoquinone indispensable to sustain the free radical chain reaction. This inhibitory effect is relaxed when the enzyme concentration becomes sufficiently low, which finally leads to a slightly faster autocatalytic reaction. Considering the importance of the enzyme concentration on the nonspecific response, we reproduced the autocatalysis experiments at $\mathrm{pH} 8.5$ with a DI concentration 100 times lower, i.e. $0.1 \mathrm{nM}$. The results in Figure 6 show a significant gain in the $\mathrm{H}_{2} \mathrm{O}_{2}$ detection limit, allowing now to detect as low as $15 \mathrm{nM} \mathrm{H}_{2} \mathrm{O}_{2}$. Compared to the previous experiments performed at a higher enzyme concentration, this corresponds to a 1o-fold gain in LOD (compare Figures 3 and 6).

To illustrate the potential of the method to indirectly detect the activity of an oxidase through its $\mathrm{H}_{2} \mathrm{O}_{2}$ production, we performed the quantitative detection of glucose oxidase (GOD) under the experimental conditions used above. Indeed, in the presence of glucose, GOD catalyzes the oxidation of glucose into gluconolactone with concomitant production of $\mathrm{H}_{2} \mathrm{O}_{2}$ from catalytic reduction of dissolved $\mathrm{O}_{2}$. The results gathered in Figure $\mathrm{S}_{23}$ demonstrate the feasibility of the approach, allowing indirect quantification of GOD by autocatalytic molecular amplification down to subpicomolar concentrations.

Triggering the autocatalysis with a naphthoquinone A compelling property of the present cross-catalytic reaction scheme lies in its ability to be triggered not only by 
$\mathrm{H}_{2} \mathrm{O}_{2}$ but also any redox cycling compound (i.e., by definition a redox-active compound able in the presence of $\mathrm{O}_{2}$ and a reducing species to cycle and to generate reactive oxygen species). This makes this approach potentially attractive for the design of new sensitive bioassays. ${ }^{60,61}$ This can be for instance through the upstream production of a redox cycling compound via an enzyme or an enzymatic label, which can then trigger via one of the above boronic probes an exponential molecular amplification. With the aim to evaluate the ability of $\mathbf{P}_{3}$ to be triggered by low concentrations of a given redox cycling compound, we have investigated the autocatalysis in the presence of different concentrations of 2-amino-3-chloro-1,4-naphthoquinone (from $0.15 \mathrm{nM}$ to $0.15 \mu \mathrm{M}$ ), a compound we had previously identified as an efficient redox cycling compound. ${ }^{34}$ The results gathered in Figure 7 demonstrates particularly good analytical performances, allowing to easily detect as low as $150 \mathrm{pM}$ of 2-amino-3-chloro-1,4-NQ in less than $30 \mathrm{~min}$, with a LOD estimated close to $50 \mathrm{pM}$. As for $\mathrm{H}_{2} \mathrm{O}_{2}$, the dynamic range of concentrations is also wide, extending over nearly 3 orders of magnitude. These analytical performances are definitely much better than those obtained with $\mathrm{H}_{2} \mathrm{O}_{2}$. This asymmetry in the analytical performances

A
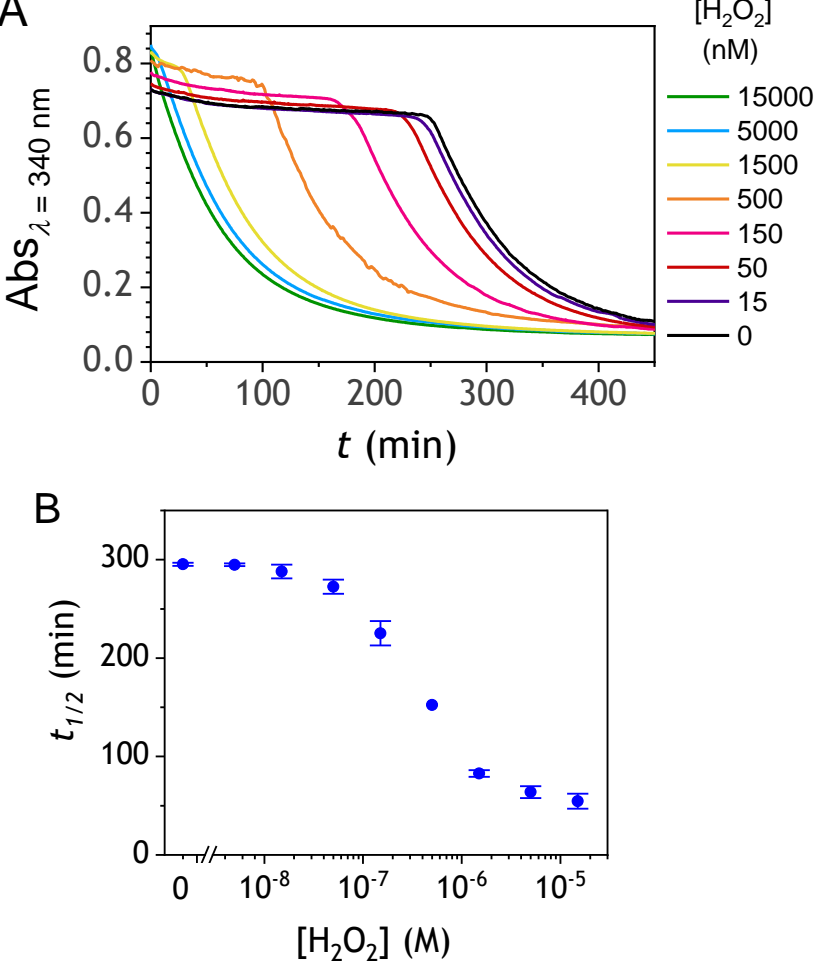

Figure 6. (A) Autocatalytic responses obtained with o.1 nM DI, $50 \mu \mathrm{M} \mathbf{P}_{3}, 250 \mu \mathrm{M}$ NADH and different $\mathrm{H}_{2} \mathrm{O}_{2}$ concentrations (see the legend on the graph) in an air-saturated Tris buffer (o.1 M) at $\mathrm{pH}$ 8.5. Each curve is the average of triplicate experiments. (B) Semi-logarithmic $\mathrm{H}_{2} \mathrm{O}_{2}$ calibration curve extracted from kinetics experiments analogous to those reported in A. Errors bars are standard deviations from triplicates. was shown to be an intrinsic property of a cross-catalytic reaction scheme. ${ }^{36}$ Indeed, if the two catalytic loops have similar rates, the detection efficiency of the catalyst of Loop 1 or Loop 2 is theoretically the same, thus leading to an identical LOD of each trigger. On the other hand, if the rates of each loop are different, the trigger of the fastest loop becomes the most easily detectable and therefore the one that theoretically leads to the lowest LOD. This is exactly what we observe here with the trigger naphthohydroquinone, which, due to its involvement in a faster catalytic loop (Loop 2) is detected more sensitively than $\mathrm{H}_{2} \mathrm{O}_{2}$.
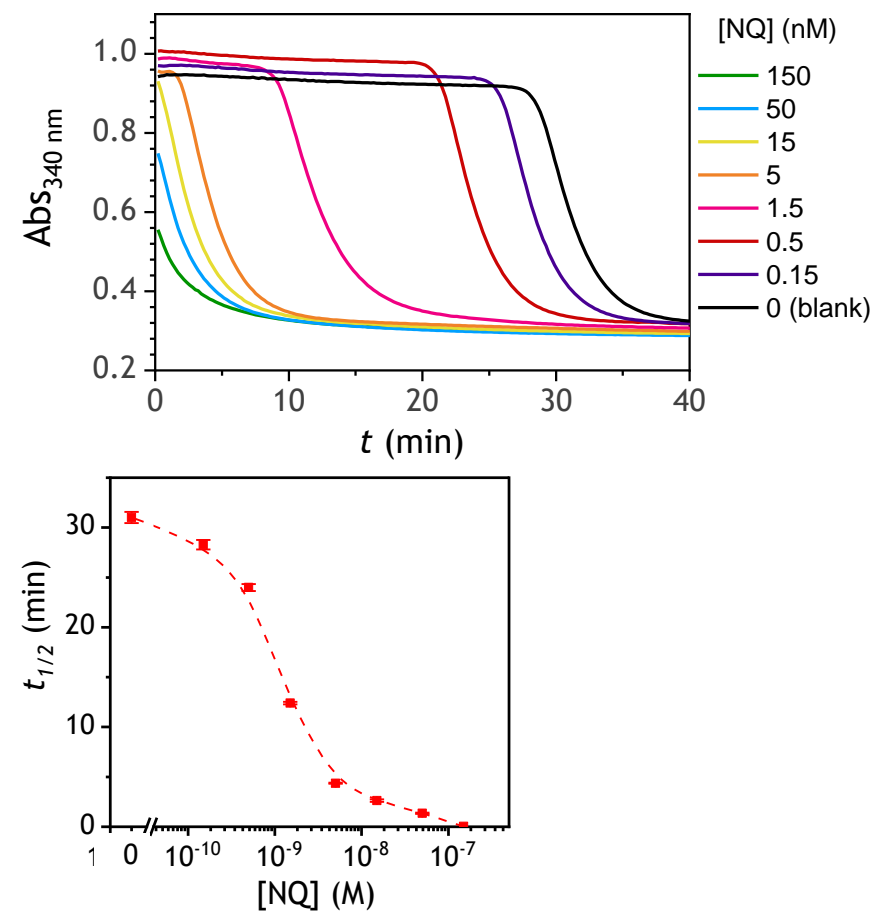

Figure 7. (Top) UV-vis kinetic traces (average of triplicates) monitored at $340 \mathrm{~nm}$ immediately after the injection of different concentrations of 2-amino-3-chloro-1,4-NQ (see the caption on the graph) to solutions containing 50 $\mu \mathrm{M} \mathbf{P}_{3}, 10 \mathrm{nM}$ DI and $250 \mu \mathrm{M}$ NADH in an air-saturated CAPS-EDTA buffer (o.1 M) at $\mathrm{pH}$ 9.5, and (bottom) the corresponding semi-logarithmic 2-amino-3-chloro-1,4-NQ calibration curve. Errors bars are standard deviations from triplicates.

Towards an improvement with the design of a diboronate probe able to release a better redox cycler

Improvement of the rates associated to each loop of the cross-catalysis reaction is a strategy to shorten the analysis time and to potentially enhance the detection limit by better discriminating the specific from nonspecific responses. ${ }^{36}$ In the present case, the background amplification stems from the instability associated to Loop 2 (i.e., from NADH autoxidation or NADH-oxidase like activity of diaphorase) since the double masking of the probe makes it so stable that no nonspecific response can arise from Loop 1. It is therefore particularly relevant to find a strategy 
that aims at improving the global rate of Loop 2. To do so, a relatively straightforward method is to substitute the masked $1,4-\mathrm{NQH}_{2}$ in $\mathbf{P}_{3}$ by an alternative faster redox cycling naphthohydroquinone. A series of different naphthoquinones were thus screened for their redox cycling properties and then compared to the 1,4-NQ (see SI and Figure S21 for details). From this screening, three naphthoquinones were identified as a better redox cycler than the 1,4$\mathrm{NQ}$, i.e. the 2-amino-3-chloro-1,4-NQ, 1,2-NQ and 2-methoxy-1,4-NQ. The chemical stability of these three naphthoquinones in an aqueous buffered solution was also examined and the most stable was the 2-methoxy-1,4-NQ, showing almost no change in the absorbance spectra after several hours in a Tris buffer (graph C in Figure S22). The chemical synthesis of the corresponding diboronic probe $\mathbf{P}_{4}$ was thus planned and successfully achieved (Scheme 1C). The long-term stability of a diluted solution of $\mathbf{P}_{4}$ was shown to be quite good (Figure $\mathrm{S}_{22}$ and $2 \mathrm{D}$ ), demonstrating a chemical stability of $\mathbf{P}_{4}$ at least as good as $\mathbf{P}_{3}$. Concerning the $\mathrm{H}_{2} \mathrm{O}_{2}$-mediated deprotection of $\mathbf{P}_{4}$ (Figure $2 \mathrm{D}$ ), it was found a little slower than $\mathbf{P}_{3}$ (by a factor $\sim 6$ ). We attribute this slower rate to the methoxy group in ortho position, which by steric effect can slow-down the nucleophilic attack of $\mathrm{HO}_{2}^{-}$.

The autocatalytic kinetic traces collected with $\mathbf{P}_{\mathbf{4}}$ over $\sim 90$ min at $\mathrm{pH} 8.5$ attest of a rather fast autocatalytic reaction (Figure 8A), approximately 4 -fold faster than $\mathbf{P}_{3}$ at the same $\mathrm{pH}$. This gain in analysis time is clearly the result of the better redox cycling properties of $2-\mathrm{MeO}-1,4-\mathrm{NQ}$ as compared to $1,4-\mathrm{NQ}$, compensating thus for the slower $\mathrm{H}_{2} \mathrm{O}_{2}$ deprotection rate. This gain in time also almost completely translates into a gain in LOD since the detection limits for $\mathbf{P}_{3}$ and $\mathbf{P}_{4}$ are 0.2 and $0.06 \mu \mathrm{M} \mathrm{H}_{2} \mathrm{O}_{2}$, respectively (Figure $8 \mathrm{C}$ ). The fact that the faster kinetics with $\mathbf{P}_{4}$ results in a better LOD is not surprising because, as we had previously demonstrated from theoretical analysis of a crosscatalytic system,,$^{36}$ reaction time and detection limit are both inversely proportional to the geometric mean of each loop's rate (this corresponds to the limiting case where detection of $\mathrm{H}_{2} \mathrm{O}_{2}$ is mainly restricted by the nonspecific response resulting from NADH autoxidation).

\section{Conclusion}

Thanks to the simple strategy consisting in the double masking of a naphthohydroquinone with boronate groups, we demonstrated the feasibility to design an efficient and versatile exponential molecular amplification system, wellsuited for the sensitive detection of $\mathrm{H}_{2} \mathrm{O}_{2}$ down to a few $\mathrm{nM}$ in less than 10 minutes or a redox cycling compound such as the 2-amino-3-chloro-1,4-NQ down to $50 \mathrm{pM}$ in less than 30 minutes. These remarkable analytical performances are the best so far reported for an autocatalytic molecular amplification strategy, amounting to a 30o-fold improvement in LOD compared to $\mathrm{H}_{2} \mathrm{O}_{2}$ detection by dendritic chain reaction. ${ }^{21}$ Such performances are the result of the high chemical stability of the diboronate ester probes (a high stability resulting from the double protection of the
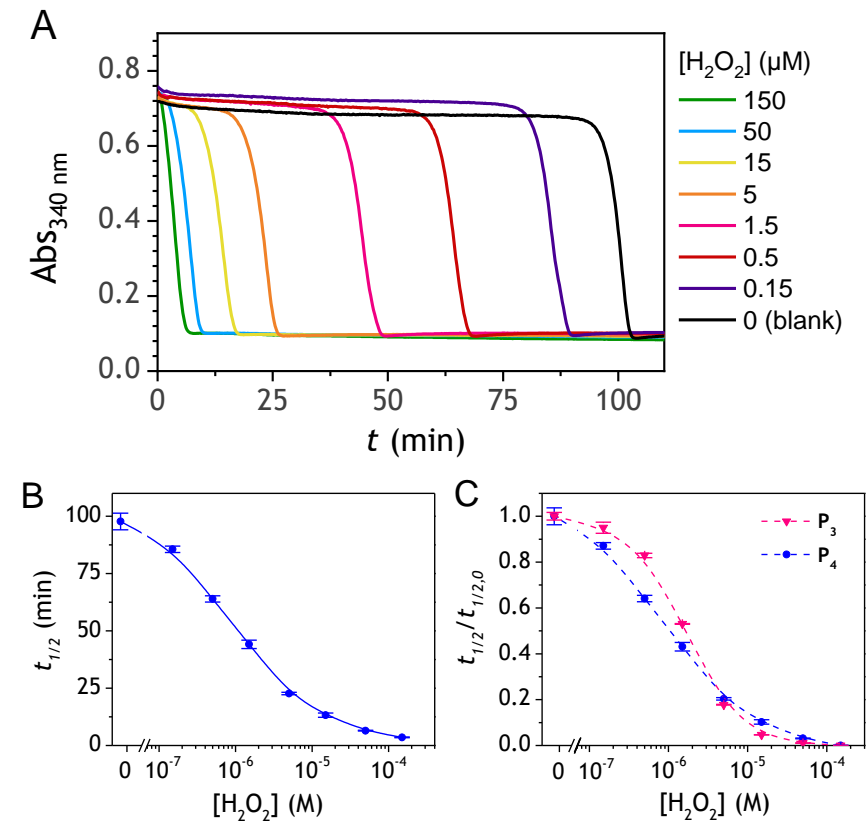

Figure 8. (Top) UV-vis kinetic traces (average of triplicates) monitored at $340 \mathrm{~nm}$ immediately after the injection of different concentrations of $\mathrm{H}_{2} \mathrm{O}_{2}$ (see the caption on the graph) to solutions containing $50 \mu \mathrm{M} \mathbf{P}_{4}, 10 \mathrm{nM}$ DI and 250 $\mu \mathrm{M}$ NADH in an air-saturated Tris-EDTA buffer (o.1 M) at $\mathrm{pH} \mathrm{8.5}$, and (bottom) the corresponding semi-logarithmic $\mathrm{H}_{2} \mathrm{O}_{2}$ calibration curve. Errors bars are standard deviations from triplicates.

probe and a non-utilization of a self-immolating protective group) as well as a particularly good reactivity towards $\mathrm{H}_{2} \mathrm{O}_{2}$. Another important result of this work is that it demonstrates the usefulness of deciphering the mechanism and kinetics of complex reaction networks in order to better understand, predict and optimize their functioning. Through this mechanistic study we were able to explain how the $\mathrm{pH}$ can play an important role and how, by adjusting it, it is possible to improve significantly the analytical performances thanks to a better discrimination of the specific $v s$. nonspecific response. It also allowed us to demonstrate that the double masking does not prevent autocatalysis from working despite the fact that two equivalents of $\mathrm{H}_{2} \mathrm{O}_{2}$ are needed to completely deprotect the probe. Furthermore, as illustrated with the selection of a better redox cycler for the design of probe $\mathbf{P}_{\mathbf{4}}$, it is clear that there is room to further improve the analytical performances of the system.

The study of complex reaction networks with highly nonlinear dynamics is a rapidly expanding field which, due to its numerous implications in the field of systems chemistry, is expected to grow rapidly in the future. Further developments and exploitations in a variety of analytical or bioanalytical applications are also expected, especially considering the versatility and generic character of the present molecular amplification strategy. Finally, we believe 
that the approach proposed here has also potential for applications in cellular imaging or drug delivery, notably by taking advantage of probes or pro-drugs capable of selectively and autocatalytically delivering the active molecule in vivo.

\section{ASSOCIATED CONTENT}

Supporting Information. Experimental Section and complementary Figures (from $\mathrm{S}_{1}$ to $\mathrm{S}_{23}$ ) on the (i) influence of $\mathrm{pH}$ on the autocatalysis with $\mathbf{P}_{2}$, (ii) UV-vis spectrophometry and HPLC characterization of probes, (iii) kinetic analysis of the $\mathrm{H}_{2} \mathrm{O}_{2}$-mediated deprotection of probes, (iv) numerical simulations of the autocatalytic reactions, $(v)$ justification of the rate constants used for the 1,4-NQ autoxidation, (vi) redox cycling experiments with the 1,4-NQ, (vii) influence of the buffer composition and $\mathrm{pH}$ on the autocatalysis with $\mathbf{P}_{3}$, (viii) screening of naphthoquinones for their redox cyling properties, and (ix) indirect detection of glucose oxidase. "This material is available free of charge via the Internet at http://pubs.acs.org."

\section{AUTHOR INFORMATION}

\section{Corresponding Authors}

mathieu.branca@u-paris.fr

francois.mavre@u-paris.fr

limoges@u-paris.fr

\section{Present Address}

† Université de Strasbourg - LSAMM, Institut Le Bel, 4, rue Blaise Pascal, 6707o Strasbourg Cedex, France.

\section{Author Contributions}

J.P. and C.R. contributed equally to this work.

\section{Funding Sources}

This work was supported by Agence Nationale de la Recherche (ANR ECOSENS project- ANR-19-CE29-0022-o).

Notes

The authors declare no competing financial interest.

\section{REFERENCES}

(1) S. Goggins, C. G. Frost, Approaches towards molecular amplification for sensing. Analyst, 2016, 141, 3157-218.

(2) P. Scrimin, L. J. Prins, Sensing through signal amplification. Chem. Soc. Rev., 2011, 40, 4488-505.

(3) B. Limoges, D. Marchal, F. Mavré, J-M. Savéant, B. Schöllhorn. Theory and Practice of Enzyme Bioaffinity Electrodes. Direct Electrochemical Product Detection. J. Am. Chem. Soc., 2008, 130, 7259-75.

(4) Engvall E. The ELISA, enzyme-linked immunosorbent assay. Clin. Chem., 2010, 56, 319-20.

(5) Konstantinou G. N. Enzyme-Linked Immunosorbent Assay (ELISA). Methods Mol. Biol., 2017, 1592, 79-94.

(6) R. Duan, X. Zuo, S. Wang, X. Quan, D. Chen, Z. Chen, L. Jiang, C. Fan, F. Xia, Lab in a Tube: Ultrasensitive Detection of MicroRNAs at the Single-Cell Level and in Breast Cancer Patients Using Quadratic Isothermal Amplification. J. Am. Chem. Soc., 2013, 135, 4604-7.

(7) S. Goggins, B. J. Marsh, A. T. Lubben and C. G. Frost, Signal transduction and amplification through enzyme-triggered ligand release and accelerated catalysis, Chem. Sci., 2015, $6,4978-85$.
(8) Z. Gao, L. Hou, M. Xu and D. Tang, Enhanced colorimetric immunoassay accompanying with enzyme cascade amplification strategy for ultrasensitive detection of low-abundance protein, Sci. Rep., 2014, 4, 3966.

(9) Zauner, T., Berger-Hoffmann, R., Muller, K., Hoffmann, R., Zuchner, T. Highly adaptable and sensitive protease assay based on fluorescence resonance energy transfer. Anal. Chem., 2011, 83, 7356-63.

(10) B. Limoges, F. Mavré, D. Marchal, J-M. Savéant, High Amplification Rates from the Association of Two Enzymes Confined within a Nanometric Layer Immobilized on an Electrode. Modeling and Illustrating Example. J. Am. Chem. Soc., 2006, 128, 6014-15.

(11) E. Valero, R. Varón, F. García-Carmona, Kinetic Analysis of a Model for Double Substrate Cycling: Highly Amplified ADP (and/or ATP) Quantification. Biophys. J., 2004, 86, 3598-606.

(12) A. J. Bisnette, S. P. Fletcher, Mechanisms of Autocatalysis. Angew. Chem. Int., 2013, 52, 12800-26.

(13) A. I. Hanopolskyi, V. A. Smaliak, A. I. Novichkov, S. N. Semenov, Autocatalysis: Kinetics, Mechanisms and Design. ChemSystemsChem, 2020, 2, e2000026.

(14) R. Plasson, A. Brandenburg, L. Jullien, H. Bersini, Autocatalyses. J. Phys. Chem. A, 2011, 115, 8073-85.

(15) J. Wilhelm, A. Pingoud, Real-Time Polymerase Chain Reaction. ChemBioChem, 2003, 4, 1120-8.

(16) J. V. Ness, L. K. V. Ness, D. J. Galas, Isothermal reactions for the amplification of oligonucleotides. PNAS, 2003, 100, 4504-9.

(17) G. Urtel, M. Van Der Hofstadt, J.-C. Galas, A. EstevezTorres, rEXPAR: An Isothermal Amplification Scheme That Is Robust to Autocatalytic Parasites. Biochemistry, 2019, 58, 2675-81.

(18) K. Shi, S. Xie, R. Tian, S. Wang, Q. Lu, D. Gao, C. Lei, H. Zhu, Z. Nie, A CRISPR-Cas autocatalysis-driven feedback amplification network for supersensitive DNA diagnostics. Sci. Adv., 2021, 7, eabc7802.

(19) G. Gines, R. Menezes, K. Nara, A.-S. Kirstetter, V. Taly, Y. Rondelez, Isothermal digital detection of microRNAs using background-free molecular circuit. $S c i . A d v ., 2020,6$, eaay5952.

(20) E. Sella, D. Shabat, Dendritic Chain Reaction. J. Am. Chem. Soc., 2009, 131, 9934-6.

(21) M. E. Roth, O. Green, S. Gnaim, D. Shabat, Dendritic, Oligomeric, and Polymeric Self-Immolative Molecular Amplification, Chem. Rev., 2016, 116, 1309-52.

(22) N. Karton-Lifshin, D. Shabat, Exponential diagnostic signal amplification via dendritic chain reaction: the dendritic effect of a self-immolative amplifier component. New J. Chem., 2012, 36, 386-93.

(23) E. Sella, A. Lubelski, J. Klafter, D. Shabat, Two-Component Dendritic Chain Reactions: Experiment and Theory. J. Am. Chem. Soc., 2010, 132, 3945-52.

(24) M. S. Baker, S. T. Phillips, A small molecule sensor for fluoride based on an autoinductive, colorimetric signal amplification reaction. Org. Biomol. Chem., 2012, 10, 3595-9.

(25) X. Sun, S. D. Dahlhauser, E. V. Anslyn, New Autoinductive Cascade for the Optical Sensing of Fluoride: Application in the Detection of Phosphoryl Fluoride Nerve Agents. J. Am. Chem. Soc., 2017, 139, 4635-8.

(26) J.-A. Gu, V. Mani, S.-T. Huang, Design and synthesis of ultrasensitive off-on fluoride detecting fluorescence probe via autoinductive signal amplification. Analyst, 2015, 140, 346-52. 
(27) S. Hisamatsu, S. Suzuki, S. Kohmoto, K. Kishikawa, Y. Yamamoto, R. Motokawa, T. Yaita. A chemiluminescence sensor with signal amplification based on a self-immolative reaction for the detection of fluoride ion at low concentrations. Tetrahedron, 2017, 73, 3993-8.

(28) S. N. Semenov, L. J. Kraft, A. Ainla, M. Zhao, M. Baghbanzadeh, V. E. Campbell, K. Kang, J. M. Fox, G. M. Whitesides, Autocatalytic, bistable, oscillatory networks of biologically relevant organic reactions. Nature, 2016, 537, 656-6o.

(29) X. Sun, E. V. Anslyn, An Auto-Inductive Cascade for the Optical Sensing of Thiols in Aqueous Media: Application in the Detection of a VX Nerve Agent Mimic. Angew. Chem. Int. Ed., 2017, 56, 9522-6.

(30) J. D. Adams, J. J. Røise, D. S. Lee, N. Murthy, The Methionase Chain Reaction: An enzyme-based autocatalytic amplification system for the detection of thiols. Chem. Commun., 2020, 56, 3175-8.

(31) J. P. Goertz, I. M. White, Peroxidyme-Amplified Radical Chain Reaction (PARCR): Visible Detection of a Catalytic Reporter. Angew. Chem. Int. Ed., 2017, 56, 13411-5.

(32) S. Kim, A. M. Dibildox, A. Aguirre-Soto, H. D. Sikes, Exponential Amplification Using Photoredox Autocatalysis. J. Am. Chem. Soc., 2021, 143, 11544-53.

(33) X. Sun, D. Shabat, S. T. Phillips, E. V. Anslyn, Self propagating amplification reactions for molecular detection and signal amplification: Advantages, pitfalls, and challenges. J. Phys. Org. Chem., 2018, e3827.

(34) J. Pallu, C. Rabin, G. Creste, M. Branca, F. Mavré, B. Limoges, Exponential molecular amplification by $\mathrm{H}_{2} \mathrm{O}_{2}$-mediated autocatalytic deprotection of boronic ester probes to redox cyclers. Chem. Eur. J., 2019, 25, 7534-46.

(35) P. Hui, M. Branca, B. Limoges, F. Mavré, An autocatalytic organic reaction network based on cross-catalysis. Chem. Commun., 2021, ASAP.

(36) M. Branca, C. Calvet, B. Limoges, F. Mavré, Specific versus non-specific response in exponential molecular amplification from cross-catalysis: modeling the influence of background amplifications on the analytical performances. ChemPhysChem, 2021, 22, 1-12.

(37) K. Yeung, K. M. Schmid, S. T. Phillips, A thermally-stable enzyme detection assay that amplifies signal autonomously in water without assistance from biological reagents. Chem. Commun., 2013, 49, 394-6.

(38) T. Yoshii, S. Onogi, H. Shigemitsu, I. Hamachi, Chemically reactive supramolecular hydrogel coupled with a signal amplification system for enhanced analyte sensitivity. $J$. Am. Chem. Soc., 2015, 137, 3360-5.

(39) Rezende F, Brandes RP, Schröder K. Detection of Hydrogen Peroxide with Fluorescent Dyes. Antioxid Redox Signal., 2018, 29, 585-602.

(40) M. Moßhammer, M. Kühl, K. Koren, Possibilities and Challenges for Quantitative Optical Sensing of Hydrogen Peroxide. Chemosensors, 2017, 5, 28.

(41) Invitrogen, Amplex ${ }^{\circledast}$ Red Hydrogen Peroxide/Peroxidase Assay Kit, in MyQubit_Amplex_Red_Peroxide_Assay UG.pdf. 2009.

(42) B. Limoges, D. Marchal, F. Mavré, J-M. Savéant, Electrochemistry of immobilized redox enzymes: kinetic characteristics of NADH oxidation catalysis at diaphorase monolayers affinity immobilized on electrodes. J. Am. Chem. Soc., 2006, 128, 2084-92.

(43) Wu J. T., Wu L. H., Knight J. A. Stability of NADPH: effect of various factors on the kinetics of degradation. Clin.
Chem., 1986, 32, 314-9. Erratum in: Clin. Chem., 1987, 33, 724.

(44) S. Fukuzumi, M. Ishikawa, T. Tanaka, Mechanisms of photo-oxidation of NADH model compounds by oxygen. J. Chem. Soc., Perkin Trans. II, 1989, 1037-45.

(45) H. G. Kuivila, Jr. J. F. Reuwer, J. A. Mangravite, Electrophilic displacement reactions: XV. Kinetics and mechanism of the base-catalyzed protodeboronation of areneboronic acids. Canadian J. Chem., 1963, 41, 3081-90.

(46) P. A. Cox, M. Reid, A. G. Leach, A. D. Campbell, E. J. King, G. C. Lloyd-Jones, Base-catalyzed aryl- $\mathrm{B}(\mathrm{OH})_{2}$ protodeboronation revisited: from concerted proton transfer to liberation of a transient aryl anion. J. Am. Chem. Soc., 2017, 139, 13156-65.

(47) H. G. Kuivila, Electrophilic Displacement Reactions. III. Kinetics of the Reaction between Hydrogen Peroxide and Benzeneboronic Acid. J. Am. Chem. Soc., 1954, 76, 870-4.

(48) A. Sikora, J. Zielonka, M. Lopez, J. Joseph, B. Kalyanaraman, Direct oxidation of boronates by peroxynitrite: Mechanism and implications in fluorescence imaging of peroxynitrite. Free Radic. Biol. Med., 2009, 47, 1401-7.

(49) E. W. Miller, A. E. Albers, A. Pralle, E. Y. Isacoff, C. J. Chang, Boronate-Based Fluorescent Probes for Imaging Cellular Hydrogen Peroxide. J. Am. Chem. Soc., 2005, 127, 16652-9.

(5o) L. Challier, R. Miranda-Castro, D. Marchal, V. Noël, F. Mavré, B. Limoges, Kinetic Rotating Droplet Electrochemistry: A Simple and Versatile Method for Reaction Progress Kinetic Analysis in Microliter Volumes. J. Am. Chem. Soc., 2013, 135, 14215-28.

(51) C.P. Lu, C.T. Lin, C.M. Chang, S.H. Wu, L.C. Lo, Nitrophenylboronic Acids as Highly Chemoselective Probes To Detect Hydrogen Peroxide in Foods and Agricultural Products. J. Agric. Food Chem., 2011, 59, 11403-6.

(52) X. Yuan, C. J. Miller, A. N. Pham, T. D. Waite, Kinetics and mechanism of auto- and copper-catalyzed oxidation of 1,4naphthohydroquinone. Free Radic. Biol. Med., 2014, 71, 291-302.

(53) V. Roginsky, T. Barsukova, Kinetics of oxidation of hydroquinones by molecular oxygen. Effect of superoxide dismutase. J. Chem. Soc., Perkin Trans., 20oo, 2, 1575-82.

(54) Y. Song, G. R. Buettner, Thermodynamic and kinetic considerations for the reaction of semiquinone radicals to form superoxide and hydrogen peroxide. Free Radic. Biol. Med., 2010, 49, 919-62.

(55) D. M. Miller, G. R. Buettner, S. D. Aust, Transition metals as catalysts of "autoxidation" reactions. Free Radic. Biol. Med., 1990, 8, 95-108.

(56) A. Nadezhidin, H. B. Dunford. The oxidation of ascorbic acid and hydroquinone by perhydroxyl radicals. A flash photolysis study. J. Phys. Chem., 1979, 83, 1957-1961.

(57) L G Forni, R L Willson. Thiyl and phenoxyl free radicals and NADH. Direct observation of one-electron oxidation. Biochem. J., 1986, 240, 897-903.

(58) H. G. Kuivila, A. G. Armour, Electrophilic displacement reactions. 9. Effects of substituents on rates of reactions between hydrogen peroxide and benzeneboronic acid. J. Am. Chem. Soc., 1957, 79, 5659-62.

(59) https://www.nipro.co.jp/en/business/other/enzymes.html\#

(6o) K. M. Soares, N. Blackmon, T. Y. Shun, S. N. Shinde, H. K.Takyi, P. Wipf, J. S. Lazo, P. A. Johnston. Profiling the 
NIH Small Molecule Repository for compounds that generate $\mathrm{H}_{2} \mathrm{O}_{2}$ by redox cycling in reducing environments. Assay Drug. Dev. Technol., 2010, 8, 152-74.

(61) K. Yeung, K. M. Schmid, S. T. Phillips, A thermally-stable enzyme detection assay that amplifies signal autonomously in water without assistance from biological reagents. Chem. Commun., 2013, 49, 394-6. 
Table 1. Rate constants values used for the different autocatalytic kinetic simulations.

\begin{tabular}{|c|c|c|c|c|c|c|c|}
\hline & \multirow[b]{2}{*}{ Rate constant } & \multicolumn{6}{|c|}{ Simulation entry ${ }^{a}$} \\
\hline & & 1 & 2 & 3 & 4 & 5 & 6 \\
\hline \multirow{2}{*}{$\begin{array}{l}\text { Probe deprotec- } \\
\text { tion reactions }\end{array}$} & $k_{d, \mathrm{P}_{3}}\left(\mathrm{M}^{-1} \mathrm{~s}^{-1}\right)$ & 35 & 35 & 35 & 35 & 35 & / \\
\hline & $k_{d, \mathrm{P}_{2}}\left(\mathrm{M}^{-1} \mathrm{~s}^{-1}\right)$ & 300 & 300 & 300 & 300 & 300 & 300 \\
\hline \multirow{3}{*}{ Side reactions } & $k_{n}\left(\mathrm{M}^{-1} \mathrm{~s}^{-1}\right)$ & 1.2 & 1.2 & 0.0 & 0.0 & 0.0 & 0.25 \\
\hline & $k_{p}\left(\mathrm{~s}^{-1}\right)$ & 0.0004 & 0.0004 & 0.0004 & 0.0004 & 0.0004 & 0.0004 \\
\hline & $k_{n s}\left(\mathrm{M}^{-1} \mathrm{~s}^{-1}\right)$ & & $10^{-4}$ & $2.75 \times 10^{-3}$ & $4.1 \times 10^{-3}$ & $4.1 \times 10^{-3}$ & $4.1 \times 10^{-3}$ \\
\hline \multirow{3}{*}{$\begin{array}{l}\text { Enzyme reac- } \\
\text { tions }\end{array}$} & $K_{\mathrm{M}}(\mu \mathrm{M})$ & 60 & 60 & 60 & 60 & 60 & 60 \\
\hline & $k_{c}\left(\mathrm{~s}^{-1}\right)$ & 1200 & 1200 & 1200 & 1200 & 1200 & 1200 \\
\hline & $k_{\text {red }}\left(\mathrm{M}^{-1} \mathrm{~s}^{-1}\right)$ & $1.5 \times 10^{9}$ & $1.5 \times 10^{9}$ & $1.5 \times 10^{9}$ & $10^{8}$ & $10^{8}$ & $10^{8}$ \\
\hline \multirow{7}{*}{$\begin{array}{l}1,4-\mathrm{NQH}_{2} \text { autoxi- } \\
\text { dation reactions }\end{array}$} & $k_{o x}\left(\mathrm{M}^{-1} \mathrm{~s}^{-1}\right)$ & 3300 & l & / & / & / & / \\
\hline & $k_{\mathrm{o}}\left(\mathrm{M}^{-1} \mathrm{~s}^{-1}\right)$ & & 0.001 & 0.001 & 0.001 & 0.001 & 0.001 \\
\hline & $\underset{c o, 1}{k_{c o}, k_{\mathrm{dis},-1}\left(\mathrm{M}^{-1}\right.}$ & & $66000 ; 10^{8}$ & $66000 ; 10^{8}$ & $66000 ; 10^{8}$ & $6.6 \times 10^{6} ; 10^{8}$ & $66000 ; 10^{8}$ \\
\hline & $k_{2}, k_{-2}\left(\mathrm{M}^{-1} \mathrm{~s}^{-1}\right)$ & & $\begin{array}{l}4.4 \times 10^{7} ; 2 \times \\
10^{8}\end{array}$ & $\begin{array}{l}4.4 \times 10^{7} ; 2 \times \\
10^{8}\end{array}$ & $\begin{array}{l}4.4 \times 10^{7} ; 2 \times \\
10^{8}\end{array}$ & $\begin{array}{l}4.4 \times 10^{7} ; 2 \times \\
10^{8}\end{array}$ & $\begin{array}{l}4.4 \times 10^{7} ; 2 \times \\
10^{8}\end{array}$ \\
\hline & $k_{3}\left(\mathrm{M}^{-1} \mathrm{~s}^{-1}\right)$ & & $8 \times 10^{4}$ & $8 \times 10^{4}$ & $5 \times 10^{4}$ & $4.7 \times 10^{6}$ & $5 \times 10^{4}$ \\
\hline & $k_{4}\left(\mathrm{M}^{-1} \mathrm{~s}^{-1}\right)$ & & $2 \times 10^{4}$ & $2 \times 10^{4}$ & $2 \times 10^{4}$ & $2 \times 10^{4}$ & $2 \times 10^{4}$ \\
\hline & $k_{5}\left(\mathrm{M}^{-1} \mathrm{~s}^{-1}\right)$ & & $2 \times 10^{9}$ & $2 \times 10^{9}$ & $2 \times 10^{9}$ & $2 \times 10^{9}$ & $2 \times 10^{9}$ \\
\hline
\end{tabular}

${ }^{a}$ Are highlighted in blue the change made when going step-by-step from the first simulation entry to the last one. 